\title{
A GENERALIZED-LAGUERRE-FOURIER-HERMITE PSEUDOSPECTRAL METHOD FOR COMPUTING THE DYNAMICS OF ROTATING BOSE-EINSTEIN CONDENSATES
}

\author{
WEIZHU BAO*, HAILIANG LI ${ }^{\dagger}$, AND JIE SHEN $\ddagger$
}

\begin{abstract}
A time-splitting generalized-Laguerre-Fourier-Hermite pseudospectral method is proposed for computing the dynamics of rotating Bose-Einstein condensates (BECs) in two and three dimensions. The new numerical method is based on the following: (i) the use of a time-splitting technique for decoupling the nonlinearity; (ii) the adoption of polar coordinate in two dimensions, and resp. cylindrical coordinate in three dimensions, such that the angular rotation term becomes constant coefficient; and (iii) the construction of eigenfunctions for the linear operator by properly scale the generalized-Laguerre, Fourier and Hermite functions. The new method enjoys the following properties: (i) it is explicit, time reversible and time transverse invariant; (ii) it conserves the position density and is spectrally accurate in space and second-order or fourth-order accurate in time; and (iii) it solves the problem in the original whole space instead of in a truncated artificial computational domain. The method is also extended to solve the coupled Gross-Pitaevskii equations for the dynamics of rotating two-component and spin-1 BECs. Extensive numerical results for the dynamics of BECs are reported to demonstrate the accuracy and efficiency of the new method for rotating BECs.
\end{abstract}

Key words. Generalized-Laguerre-Fourier-Hermite functions, rotating Bose-Einstein condensate, angular momentum rotation, time-splitting, energy, condensate width.

AMS subject classifications. 35Q55, 65T99, 65Z05, 65N12, 65N35, 81-08

1. Introduction. Since the realization of Bose-Einstein condensation (BEC) of alkali atoms and hydrogen in dilute bosonic atomic gases [3, 16], much attention has been focused on its dynamical phenomena associated with superfluidity [34, 32, $18,14,2,44]$. A remarkable feature of superfluids is the appearance of quantized vortices $[34,38,1,37,33,24,28]$. In fact, quantized vortices have a long history that begins with the study of liquid Helium and superconductors. Their appearance is viewed as a typical signature of superfluidity which describes a phase of matter characterized by the complete absence of viscosity. In other words, if placed in a closed loop, superfluids can flow endlessly without friction. Different research groups have obtained quantized vortices in BEC experimentally, e.g. the JILA group [34], the ENS group [32, 33] and the MIT group [37]. Several experimental methods of vortex creation are currently in use for studying BEC, including phase imprinting [34], cooling of a rotating normal gas [21], and conversion of spin angular momentum into orbital angular momentum by reversal of the magnetic bias field in an Ioffe-Pritchard trap $[44,29,30]$. It is expected that more complicated vortex clusters can be created in the

*Department of Mathematics and Center for Computational Science and Engineering, National University of Singapore, Singapore 117543 (bao@math.nus.edu.sg, http://www.math.nus.edu.sg/ bao/). The research of this author was supported by Ministry of Education of Singapore grant No. R-158-000-002-112 and R-146-000-120-112.

$\dagger$ School of Mathematics, Capital Normal University, Northern Road 105, Western Ring 3, 100037, Beijing, P. R. China (hailiang_li2002@yahoo.com.cn). The research of this author was supported by National Natural Science Foundation of China grant No. 10431060 and Beijing Nova Program grant No. 2005B48.

$\ddagger$ Department of Mathematics, Purdue University, West Lafayette, IN 47907, USA (shen@math.purdue.edu). The research of this author was supported by NSF DMS-0610646 and AFOSR FA9550-08-1-0416. 
future, e.g. with further developments of the phase-imprinting method. Such states and their dynamics would enable various opportunities, ranging from investigating the properties of random polynomials [15] to using vortices in quantum memories [25]. The recent experimental and theoretical advances in exploration of quantized vortices in BEC have spurred great excitement in the atomic physics and computational and applied mathematics communities, and renewed interest in studying superfluidity.

In this paper, we consider a rotating Bose-Einstein condensate (BEC) in an external trapping potential $V_{t}(x, y, z)=\frac{1}{2} m_{b}\left(\omega_{x}^{2} x^{2}+\omega_{y}^{2} y^{2}+\omega_{z}^{2} z^{2}\right)+W_{t}(x, y, z)$ with $\omega_{x}, \omega_{y}$ and $\omega_{z}$ the trap frequencies in $x-, y$ - and $z$-direction, respectively, $m_{b}$ the mass of BEC atoms, and $W_{t}(x, y, z)$ a real-valued function. We assume that the interaction strength within the BEC is $U_{0}$, given by $U_{0}=4 \pi \hbar^{2} a_{s} / m_{b}$ with $a_{s}$ being the s-wave scattering length. For temperatures well below the critical temperature of the BEC, the dynamics of the rotating BEC is well described by the dimensionless Gross-Pitaevskii equation (GPE) with an angular momentum rotation term in the $d$-dimensions $(d=2,3)[36,18,14,5]$ :

$$
\begin{aligned}
& i \partial_{t} \psi(\mathbf{x}, t)=\left[-\frac{1}{2} \Delta+V(\mathbf{x})-\Omega L_{z}+\beta_{d}|\psi(\mathbf{x}, t)|^{2}\right] \psi(\mathbf{x}, t), \quad \mathbf{x} \in \mathbb{R}^{d}, t>0, \\
& \psi(\mathbf{x}, 0)=\psi_{0}(\mathbf{x}), \quad \mathbf{x} \in \mathbb{R}^{d} .
\end{aligned}
$$

Here, $\psi=\psi(\mathbf{x}, t)$ is the dimensionless wave function; $V(\mathbf{x})$ is the dimensionless external trapping potential; $\beta_{d}=\beta$ for $d=3$, and $\beta_{d}=\beta \sqrt{\gamma_{z} / 2 \pi}$ for $d=2$ with $\beta=\frac{4 \pi N a_{s}}{a_{0}}$ characterizing the inter-atomic interaction in terms of the total number of particles $N$ in the condensate, the s-scattering wave length $a_{s}$ and the dimensionless length unit $a_{0} ; \Omega$ is the dimensionless angular momentum rotation speed; $L_{z}=-i\left(x \partial_{y}-y \partial_{x}\right)=i\left(y \partial_{x}-x \partial_{y}\right)=-i \partial_{\theta}$ is the dimensionless $z$-component angular momentum with $(r, \theta, z)$ the cylindrical coordinates when $d=3$, and $(r, \theta)$ the polar coordinates when $d=2$. We split the trapping potential into two parts, i.e.

$$
V(\mathbf{x})=V_{s}(\mathbf{x})+W(\mathbf{x}), \quad \mathbf{x} \in \mathbb{R}^{d},
$$

where $V_{s}(\mathbf{x})$ is the radial and cylindrical symmetric part when $d=2$ and $d=3$, respectively,

$$
V_{s}(\mathbf{x})=\frac{1}{2} \begin{cases}\gamma_{r}^{2}\left(x^{2}+y^{2}\right)=\gamma_{r}^{2} r^{2}, & d=2, \\ \gamma_{r}^{2}\left(x^{2}+y^{2}\right)+\gamma_{z}^{2} z^{2}=\gamma_{r}^{2} r^{2}+\gamma_{z}^{2} z^{2}, & d=3,\end{cases}
$$

with $r=\sqrt{x^{2}+y^{2}}$ and $\gamma_{x}=\frac{\omega_{x}}{\omega}, \gamma_{y}=\frac{\omega_{y}}{\omega}, \gamma_{z}=\frac{\omega_{z}}{\omega}, \gamma_{r}=\min \left\{\gamma_{x}, \gamma_{y}\right\}$ with $\omega=\min \left\{\omega_{x}, \omega_{y}, \omega_{z}\right\}$; and $W(\mathbf{x})$ is the rest of the external trapping potential.

The above dimensionless quantities in three dimensions (3D) are obtained by scaling the length by the harmonic oscillator length $a_{0}=\sqrt{\hbar / \omega m_{b}}$, the time by $\omega^{-1}$ and the energy by $\hbar \omega$. In fact, the two-dimensional (2D) GPE can be viewed as a quasi-3D experimental setup with a strong confinement in the $z$-direction, i.e. $\omega_{x} \approx \omega_{y}$ and $\omega_{z} \gg \omega_{x}$ [13]. Two important invariants of (1.1) are the normalization of the wave function $[36,5]$

$$
N(\psi)=\int_{\mathbb{R}^{d}}|\psi(\mathbf{x}, t)|^{2} d \mathbf{x} \equiv \int_{\mathbb{R}^{d}}|\psi(\mathbf{x}, 0)|^{2} d \mathbf{x}=N\left(\psi_{0}\right)=1, \quad t \geq 0
$$

and the energy

$$
\begin{aligned}
E_{\beta, \Omega}(\psi) & =\int_{\mathbb{R}^{d}}\left[\frac{1}{2}|\nabla \psi|^{2}+\left[V_{s}(\mathbf{x})+W(\mathbf{x})\right]|\psi|^{2}+\frac{\beta_{d}}{2}|\psi|^{4}-\Omega \operatorname{Re}\left(\bar{\psi} L_{z} \psi\right)\right] d \mathbf{x} \\
& \equiv E_{\beta, \Omega}\left(\psi_{0}\right), \quad t \geq 0,
\end{aligned}
$$


where $\bar{f}$ and $\operatorname{Re}(f)$ denote the conjugate and the real part of the function $f$, respectively. For well-posedness and dynamical laws of the GPE (1.1), we refer to [5, 22].

In order to study effectively the dynamics of rotating BEC, it is important to design an efficient and accurate numerical method for solving the problem (1.1). For nonrotating BEC, i.e. $\Omega=0$ in (1.1), many efficient and spectrally accurate numerical methods were proposed in the literatures (cf. $[6,7,10,35]$ and the references therein). For rotating BEC, i.e. $\Omega \neq 0$ in (1.1), the angular momentum rotation term introduces new numerical difficulties which have to be properly tackled. Recently, Bao et al. [5] presented an efficient and accurate method based on the adoption of polar coordinates in 2D and cylindrical coordinates in 3D so as to make the coefficient of the angular momentum rotation term constant; Bao and Wang [12] proposed an efficient and spectrally accurate method based on properly using the alternating direction implicit (ADI) technique for the coupling of the angular momentum term. For rotating BEC in the strong repulsive interaction and/or rapid rotation regime, i.e. $\beta_{d} \gg 1$ and/or $|\Omega|=O(1)$ in (1.1), due to the appearance of quantized vortex lattices, multiscale structures appear in the solution of the $\operatorname{GPE}(1.1)[17,14,13,26]$. In this case, most of the numerical methods for rotating BEC have some drawbacks: (i) Often the original whole space is truncated to an artificial computational domain with an artificial boundary condition (usually homogeneous Dirichlet boundary conditions are used). How to choose an appropriate bounded computational domain is a difficulty task in practice: if it is too large, the computational resource is wasted; if it is too small, the boundary effect will lead to wrong numerical solutions. (ii) The method in [5] uses polar coordinates in 2D and cylindrical coordinates in 3D such that the angular momentum rotation term becomes constant coefficient, but the method is only second or fourth order accurate in the radial direction. On the other hand, the method in [12] is of spectral accuracy in space, but it decouples the angular momentum rotation term into two steps which may cause some problems in rapid rotating regime, i.e. $|\Omega| \approx 1$.

The aim of this paper is to develop a method which enjoys the combined advantages of the numerical methods in [5] and [12]. That is to say, the method is explicit and of spectral accurate in space, and it adopts polar coordinates in $2 \mathrm{D}$ and cylindrical coordinates in 3D. We shall present such an efficient and spectrally accurate numerical method for discretizing the GPE in (1.1) by applying the time-splitting technique for decoupling the nonlinearity, adopting polar coordinates in 2D and cylindrical coordinates in $3 \mathrm{D}$ such that the angular rotation term becomes constant coefficient and using the properly scaled generalized-Laguerre, Fourier and Hermite functions as spectral basis.

The paper is organized as follows. In the next section, we preset a time-splitting pseudospectral method based on the scaled generalized-Laguerre, Fourier and Hermite functions for computing the dynamics of rotating BEC in $2 \mathrm{D}$ and 3D. In section 3, the numerical method is extended to coupled Gross-Pitaevskii equations (CGPEs) for the dynamics of rotating two-component and spin-1 BEC. In section 4, we report numerical results on the dynamics of rotating BEC to demonstrate the efficiency and accuracy of our new numerical methods. Finally, some concluding remarks are drawn in Section 5.

2. Time-splitting generalized-Laguerre-Fourier-Hermite pseudospectral method. In this section we present a second-order time-splitting generalized-LaguerreFourier-Hermite pseudospectral method for the problem (1.1) in 2D and 3D by using polar and cylindrical coordinates, respectively. 
2.1. Time-splitting. Denoting

$$
\begin{aligned}
B_{\perp} \phi & =\left[-\frac{1}{2}\left(\frac{\partial^{2}}{\partial x^{2}}+\frac{\partial^{2}}{\partial y^{2}}\right)+\frac{1}{2} \gamma_{r}^{2}\left(x^{2}+y^{2}\right)-\Omega L_{z}\right] \phi, \\
B_{z} \phi & =\left[-\frac{1}{2} \frac{\partial^{2}}{\partial z^{2}}+\frac{1}{2} \gamma_{z}^{2} z^{2}\right] \phi, \\
A \phi & =\left[W(\mathbf{x})+\beta_{d}|\phi|^{2}\right] \phi, \quad B \phi= \begin{cases}B_{\perp} \phi & d=2, \\
\left(B_{\perp}+B_{z}\right) \phi & d=3,\end{cases}
\end{aligned}
$$

then the GPE in (1.1) becomes

$$
i \partial_{t} \psi(\mathbf{x}, t)=A \psi+B \psi, \quad \mathbf{x} \in \mathbb{R}^{d}, \quad t>0 .
$$

For a given time step $\Delta t>0$, let $t_{n}=n \Delta t, n=0,1,2, \ldots$, and $\psi^{n}:=\psi^{n}(\mathbf{x})$ be the approximation of $\psi\left(\mathbf{x}, t_{n}\right)$. A second-order symplectic time integrator $[41,6,7]$ for (2.4) is as follows

$$
\psi^{(1)}=e^{-i \frac{\Delta t}{2} A} \psi^{n}, \quad \psi^{(2)}=e^{-i \Delta t B} \psi^{(1)}, \quad \psi^{n+1}=e^{-i \frac{\Delta t}{2} A} \psi^{(2)} .
$$

Thus the key for an efficient implementation of (2.5) is to solve efficiently the following two subproblems:

$$
i \partial_{t} \psi(\mathbf{x}, t)=A \psi(\mathbf{x}, t)=\left[W(\mathbf{x})+\beta_{d}|\psi(\mathbf{x}, t)|^{2}\right] \psi(\mathbf{x}, t), \quad \mathbf{x} \in \mathbb{R}^{d},
$$

and

$$
\begin{aligned}
& i \partial_{t} \psi(\mathbf{x}, t)=B \psi(\mathbf{x}, t)=\left[-\frac{1}{2} \Delta+V_{s}(\mathbf{x})-\Omega L_{z}\right] \psi(\mathbf{x}, t), \quad \mathbf{x} \in \mathbb{R}^{d}, \\
& \lim _{|\mathbf{x}| \rightarrow+\infty} \psi(\mathbf{x}, t)=0 .
\end{aligned}
$$

The decaying condition in (2.7) is due to the external trapping potential and it is necessary for satisfying the normalization (1.4).

Multiplying (2.6) by $\overline{\psi(\mathbf{x}, t)}$, we find that the ODE (2.6) leaves $|\psi(\mathbf{x}, t)|$ invariant in time $t[6,7]$. Hence for $t \geq t_{s}\left(t_{s}\right.$ is any given time), (2.6) becomes

$$
i \partial_{t} \psi(\mathbf{x}, t)=\left[W(\mathbf{x})+\beta_{d}\left|\psi\left(\mathbf{x}, t_{s}\right)\right|^{2}\right] \psi(\mathbf{x}, t), \quad t \geq t_{s}, \quad \mathbf{x} \in \mathbb{R}^{d},
$$

which can be integrated exactly, i.e.

$$
\psi(\mathbf{x}, t)=e^{-i\left[W(\mathbf{x})+\beta_{d}\left|\psi\left(\mathbf{x}, t_{s}\right)\right|^{2}\right]\left(t-t_{s}\right)} \psi\left(\mathbf{x}, t_{s}\right), \quad t \geq t_{s}, \quad \mathbf{x} \in \mathbb{R}^{d} .
$$

Thus, it remains to find an efficient and accurate scheme for (2.7). Since $B$ is a linear operator, it is most convenient to use its eigenfunctions as spectral basis functions. Thanks to (2.3), we only need to find eigenfunctions of the linear operators $B_{\perp}$ and $B_{z}$. Below we shall construct suitable spectral basis functions by properly scale the Hermite functions, generalized Laguerre functions and Fourier series which are eigenfunction of $B$ so that $e^{-i \Delta t B} \psi$ can be exactly evaluated in phase space, which is necessary for the final scheme to be time reversible and time transverse invariant. Here, the only time discretization error of the corresponding time-splitting method (2.5) is the splitting error, which is second-order in $\Delta t$. Furthermore, the scheme is explicit, time reversible, and time transverse invariant, and as we shall show below, it also conserves the normalization in time discretization.

REMARK 2.1. It is straightforward to design high-order, e.g. fourth-order, symplectic time integrator for $(2.4)[45,10,43]$. The details are omitted here for brevity. 
2.2. Generalized-Laguerre-Fourier pseudospectral method for rotating BEC in 2D. In the $2 \mathrm{D}$ case, we use the polar coordinates $(r, \theta)$, and write the solutions of $(2.7)$ as $\psi(r, \theta, t)$. Therefore, for $t \geq t_{s}$ ( $t_{s}$ is any given time), (2.7) collapses to

$$
\begin{aligned}
& i \partial_{t} \psi(r, \theta, t)=\left[-\frac{1}{2 r} \frac{\partial}{\partial r}\left(r \frac{\partial}{\partial r}\right)-\frac{1}{2 r^{2}} \frac{\partial^{2}}{\partial \theta^{2}}+\frac{1}{2} \gamma_{r}^{2} r^{2}+i \Omega \partial_{\theta}\right] \psi(r, \theta, t) \\
& :=B_{\perp} \psi(r, \theta, t), \\
& \psi(r, \theta+2 \pi, t)=\psi(r, \theta, t), \quad 0<r<\infty, \quad 0<\theta<2 \pi \\
& \lim _{r \rightarrow \infty} \psi(r, \theta, t)=0 .
\end{aligned}
$$

The normalization (1.4) collapses to

$$
\|\psi(\cdot, t)\|^{2}=\int_{0}^{\infty} \int_{0}^{2 \pi}|\psi(r, \theta, t)|^{2} r d r d \theta=\int_{0}^{\infty} \int_{0}^{2 \pi}\left|\psi_{0}(r, \theta)\right|^{2} r d r d \theta=1 .
$$

Note that it can be shown, similarly as for the Poisson equation in a $2 \mathrm{D}$ disk $[39,19$, $20]$, that the problem (2.10) admits a unique solution without any condition at the pole $r=0$.

We now construct the eigenfunctions of the linear operator $B_{\perp}$ in (2.10). For any fixed $m(m=0, \pm 1, \pm 2, \ldots)$ and $g(r)$, we have

$$
\begin{aligned}
B_{\perp}\left(g(r) e^{i m \theta}\right) & =\left[-\frac{1}{2 r} \frac{\partial}{\partial r}\left(r \frac{\partial}{\partial r}\right)-\frac{1}{2 r^{2}} \frac{\partial^{2}}{\partial \theta^{2}}+\frac{1}{2} \gamma_{r}^{2} r^{2}+i \Omega \partial_{\theta}\right]\left(g(r) e^{i m \theta}\right) \\
& =e^{i m \theta}\left[-\frac{1}{2 r} \frac{d}{d r}\left(r \frac{d}{d r}\right)+\frac{m^{2}}{2 r^{2}}+\frac{1}{2} \gamma_{r}^{2} r^{2}-m \Omega\right] g(r) \\
& :=B_{r}^{|m|}(g(r)) e^{i m \theta}-m \Omega g(r) e^{i m \theta},
\end{aligned}
$$

where

$$
B_{r}^{|m|} g(r)=\left[-\frac{1}{2 r} \frac{d}{d r}\left(r \frac{d}{d r}\right)+\frac{|m|^{2}}{2 r^{2}}+\frac{1}{2} \gamma_{r}^{2} r^{2}\right] g(r) .
$$

This immediately suggests us to construct eigenfunctions of the linear operator $B_{r}^{m}$ for any fixed $m(m=0,1,2, \ldots)$. To this end, we recall below the definition and properties of the generalized Laguerre polynomials.

For any fixed $m(m=0,1,2, \ldots)$, let $\hat{L}_{k}^{m}(r)(k=0,1,2, \ldots)$ be the generalizedLaguerre polynomials of degree $k$ satisfying $[40,11,19,20]$

$$
\begin{aligned}
& \left(r \frac{d^{2}}{d r^{2}}+(m+1-r) \frac{d}{d r}\right) \hat{L}_{k}^{m}(r)+k \hat{L}_{k}^{m}(r)=0, \quad k=0,1,2, \ldots \\
& \int_{0}^{\infty} r^{m} e^{-r} \hat{L}_{k}^{m}(r) \hat{L}_{k^{\prime}}^{m}(r) d r=C_{k}^{m} \delta_{k k^{\prime}}, \quad k, k^{\prime}=0,1,2, \ldots
\end{aligned}
$$

where $\delta_{k k^{\prime}}$ is the Kronecker delta and

$$
C_{k}^{m}=\Gamma(m+1)\left(\begin{array}{c}
k+m \\
k
\end{array}\right)=\prod_{j=1}^{m}(k+j), \quad k=0,1,2, \ldots
$$


We define the scaled generalized-Laguerre functions $L_{k}^{m}$ by

$$
L_{k}^{m}(r)=\frac{\gamma_{r}^{(m+1) / 2}}{\sqrt{\pi C_{k}^{m}}} r^{m} e^{-\gamma_{r} r^{2} / 2} \hat{L}_{k}^{m}\left(\gamma_{r} r^{2}\right) .
$$

Plugging (2.16) into (2.14) and (2.15), a tedious but simple computation [11,10] leads to

$$
\begin{aligned}
B_{r}^{m} L_{k}^{m}(r) & =\left[-\frac{1}{2 r} \frac{d}{d r}\left(r \frac{d}{d r}\right)+\frac{m^{2}}{2 r^{2}}+\frac{1}{2} \gamma_{r}^{2} r^{2}\right] L_{k}^{m}(r) \\
& =\left[\gamma_{r}(2 k+m+1)\right] L_{k}^{m}(r) \\
& 2 \pi \int_{0}^{\infty} L_{k}^{m}(r) L_{k^{\prime}}^{m}(r) r d r=\delta_{k k^{\prime}}
\end{aligned}
$$

Hence $\left\{L_{k}^{m}\right\}_{k=0}^{\infty}$ are eigenfunctions of the linear operator $B_{r}^{m}$.

For any fixed $m(m=0, \pm 1, \pm 2, \ldots)$, we derive from the above that

$$
\begin{aligned}
B_{\perp}\left(L_{k}^{|m|}(r) e^{i m \theta}\right) & =\left(B_{r}^{|m|} L_{k}^{|m|}(r)\right) e^{i m \theta}-m \Omega L_{k}^{|m|}(r) e^{i m \theta} \\
& =\left[\gamma_{r}(2 k+|m|+1)-m \Omega\right]\left(L_{k}^{|m|}(r) e^{i m \theta}\right) \\
& =\mu_{k m} L_{k}^{|m|}(r) e^{i m \theta}, \quad k=0,1,2, \ldots
\end{aligned}
$$

where

$$
\mu_{k m}=\gamma_{r}(2 k+|m|+1)-m \Omega, \quad k=0,1,2, \ldots
$$

This immediately implies that $\left\{L_{k}^{|m|}(r) e^{i m \theta}, k=0,1, \cdots, m=0, \pm 1, \pm 2, \cdots\right\}$ are eigenfunctions of the linear operator $B_{\perp}$.

For fixed even integer $M>0$ and integer $K>0$, let $X_{K M}=\operatorname{span}\left\{L_{k}^{|m|}(r) e^{i m \theta}\right.$ : $k=0,1, \ldots, K, m=-M / 2,-M / 2+1, \ldots,-1,0,1, \ldots, M / 2-1\}$. The generalizedLaguerre-Fourier spectral method for $(2.10)$ is to find $\psi_{K M}(r, \theta, t) \in X_{K M}$, i.e.

$$
\psi_{K M}(r, \theta, t)=\sum_{m=-M / 2}^{M / 2-1}\left[e^{i m \theta} \sum_{k=0}^{K} \hat{\psi}_{k m}(t) L_{k}^{|m|}(r)\right], \quad 0 \leq r<\infty, 0 \leq \theta \leq 2 \pi
$$

such that

$$
\begin{aligned}
i \frac{\partial \psi_{K M}(r, \theta, t)}{\partial t} & =\left[-\frac{1}{2 r} \frac{\partial}{\partial r}\left(r \frac{\partial}{\partial r}\right)-\frac{1}{2 r^{2}} \frac{\partial^{2}}{\partial \theta^{2}}+\frac{1}{2} \gamma_{r}^{2} r^{2}+i \Omega \partial_{\theta}\right] \psi(r, \theta, t) \\
& =B_{\perp} \psi_{K M}(r, \theta, t), \quad 0<r<\infty, \quad 0<\theta<2 \pi .
\end{aligned}
$$

Noting that $\lim _{r \rightarrow \infty} L_{k}^{|m|}(r)=0$ for $k=0,1,2, \ldots$ and $m=0, \pm 1, \pm 2, \ldots$ [40]; hence, $\lim _{r \rightarrow \infty} \psi_{K M}(r, \theta, t)=0$ is automatically satisfied. In addition, the expansions in $r$ - and $\theta$-directions for (2.21) don't commute. Plugging (2.21) into (2.22), thanks to (2.19), noticing the orthogonality of the Fourier series, for $k=0,1, \ldots, K$ and $m=-M / 2,-M / 2-1, \ldots,-1,0,1, \ldots, M / 2-1$, we find

$$
i \frac{d \hat{\psi}_{k m}(t)}{d t}=\mu_{k m} \hat{\psi}_{k m}(t)=\left[\gamma_{r}(2 k+|m|+1)-m \Omega\right] \hat{\psi}_{k m}(t)
$$


The above linear ODE can be integrated exactly and the solution is given by

$$
\hat{\psi}_{k m}(t)=e^{-i \mu_{k m}\left(t-t_{s}\right)} \hat{\psi}_{k m}\left(t_{s}\right), \quad t \geq t_{s} .
$$

Plugging (2.24) into (2.21), we obtain the solution of (2.22) as

$$
\begin{aligned}
\psi_{K M}(r, \theta, t) & =e^{-i\left(t-t_{s}\right) B_{\perp}} \psi_{K M}\left(r, \theta, t_{s}\right) \\
& =\sum_{m=-M / 2}^{M / 2-1}\left[e^{i m \theta} \sum_{k=0}^{K} e^{-i \mu_{k m}\left(t-t_{s}\right)} \hat{\psi}_{k m}\left(t_{s}\right) L_{k}^{|m|}(r)\right], \quad t \geq t_{s},
\end{aligned}
$$

with

$$
\hat{\psi}_{k m}\left(t_{s}\right)=\frac{1}{2 \pi} \int_{0}^{2 \pi}\left[e^{-i m \theta} \int_{0}^{\infty} \psi_{K M}\left(r, \theta, t_{s}\right) L_{k}^{|m|}(r) r d r\right] d \theta .
$$

The above procedure is not suitable in practice due to the difficulty to compute the integrals in (2.26). We now present an efficient implementation by choosing $\psi_{K M}^{0}(r, \theta)$ as the interpolation of $\psi(r, \theta, 0)$ on a suitable grid, and approximating (2.26) (for all $m$ ) by a quadrature rule on this grid.

For each fixed $m$, it is clear that the optimal quadrature rule, hence the collocation points, for the $r$-integral in (2.26) depends on $m$ [11]. Since we need take the Fourier transform in the azimuthal direction too, thus, we have to use the same set of collocation points for all $m$ to form a tensorial grid in the $(r, \theta)$ domain. Therefore, let $\left\{\hat{r}_{j}\right\}_{j=0}^{K+M / 2}$ be the Laguerre-Gauss points [40,39]; i.e. they are the $K+M / 2+1$ roots of the standard Laguerre polynomial $\hat{L}_{K+M / 2+1}^{0}(r):=\hat{L}_{K+M / 2+1}(r)$. Let $\left\{\hat{\omega}_{j}\right\}_{j=0}^{K+M / 2}$ be the corresponding weights associated with the generalized-Laguerre-Gauss quadrature $[40,39]$; i.e., we have

$$
\int_{0}^{\infty} f(r) e^{-r} d r=\sum_{j=0}^{K+M / 2} f\left(\hat{r}_{j}\right) \hat{\omega}_{j}, \quad \forall f \in P_{2 K+M+1},
$$

where $P_{2 K+M+1}$ are the space of polynomials of degree less or equal than $2 K+M+1$. Hence, for $k, k^{\prime}=0,1, \ldots, K,|m| \leq M / 2$, we have

$$
\sum_{j=0}^{K+M / 2} \hat{\omega}_{j}\left(\hat{r}_{j}\right)^{m} \frac{\hat{L}_{k}^{m}\left(\hat{r}_{j}\right)}{\sqrt{C_{k}^{m}}} \frac{\hat{L}_{k^{\prime}}^{m}\left(\hat{r}_{j}\right)}{\sqrt{C_{k^{\prime}}^{m}}}=\int_{0}^{\infty} r^{m} e^{-r} \frac{\hat{L}_{k}^{m}(r)}{\sqrt{C_{k}^{m}}} \frac{\hat{L}_{k^{\prime}}^{m}(r)}{\sqrt{C_{k^{\prime}}^{m}}} d r=\delta_{k k^{\prime}}
$$

We then define the scaled generalized-Laguerre-Gauss points and weights by

$$
r_{j}=\sqrt{\frac{\hat{r}_{j}}{\gamma_{r}}}, \quad \omega_{j}=\frac{\pi \hat{\omega}_{j} e^{\hat{r}_{j}}}{\gamma_{r}}, \quad j=0,1, \ldots, K+M / 2 .
$$

We derive from (2.16) and (2.28) that

$$
\begin{aligned}
\sum_{j=0}^{K+M / 2} \omega_{j} L_{k}^{m}\left(r_{j}\right) L_{k^{\prime}}^{m}\left(r_{j}\right) & =\sum_{j=0}^{K+M / 2} \frac{\pi \hat{\omega}_{j} e^{\hat{r}_{j}}}{\gamma_{r}} L_{k}^{m}\left(\sqrt{\hat{r}_{j} / \gamma_{r}}\right) L_{k^{\prime}}^{m}\left(\sqrt{\hat{r}_{j} / \gamma_{r}}\right) \\
& =\sum_{j=0}^{K+M / 2} \hat{\omega}_{j}\left(\hat{r}_{j}\right)^{m} \frac{\hat{L}_{k}^{m}\left(\hat{r}_{j}\right)}{\sqrt{C_{k}^{m}}} \frac{\hat{L}_{k^{\prime}}^{m}\left(\hat{r}_{j}\right)}{\sqrt{C_{k^{\prime}}^{m}}} \\
& =\delta_{k k^{\prime}}, \quad k, k^{\prime}=0,1, \ldots, K,|m| \leq M / 2 .
\end{aligned}
$$


Note that the computation of the weights $\left\{\omega_{j}\right\}$ from (2.29) is not a stable process for large $K$ and $M$. However, they can be computed in a stable way as suggested in the Appendix of [39].

Let $\theta_{s}=\frac{2 s \pi}{M}(s=0,1, \cdots, M-1)$. For any given set of values $\left\{\psi_{j s}, 0 \leq j \leq\right.$ $K+M / 2 ; 0 \leq s \leq M-1\}$, we can define a unique function $\psi$ in $X_{K M}$ interpolating this set, i.e.,

$$
\begin{aligned}
& \psi(r, \theta)=\sum_{m=-M / 2}^{M / 2-1} \sum_{k=0}^{K} \widehat{\psi}_{k m} L_{k}^{|m|}(r) e^{i m \theta} \text { such that } \\
& \psi\left(r_{j}, \theta_{s}\right)=\psi_{j s}, \quad 0 \leq j \leq K+M / 2 ; 0 \leq s \leq M-1 .
\end{aligned}
$$

By using the discrete orthogolarity relation (2.30) for the scaled generalized Laguerre functions and the discrete Fourier orthogolarity relation

$$
\frac{1}{M} \sum_{s=0}^{M-1} e^{i k \theta_{s}} e^{-i k^{\prime} \theta_{s}}=\delta_{k, k^{\prime}}, \quad|k| \leq M / 2
$$

we find that

$$
\widehat{\psi}_{k m}=\frac{1}{M} \sum_{s=0}^{M-1}\left[e^{-i m \theta_{s}} \sum_{j=0}^{K+M / 2} \omega_{j} \psi_{j s} L_{k}^{|m|}\left(r_{j}\right)\right]
$$

and that

$$
\|\psi\|^{2}:=\int_{0}^{2 \pi} \int_{0}^{\infty}|\psi|^{2} r d r d \theta=2 \pi \sum_{m=-M / 2}^{M / 2-1} \sum_{k=0}^{K}\left|\widehat{\psi}_{k m}\right|^{2}=\frac{2 \pi}{M} \sum_{j=0}^{K+M / 2} \sum_{s=0}^{M-1}\left|\psi_{j s}\right|^{2} \omega_{j} .
$$

We can now describe the second-order time-splitting generalized-Laguerre-Fourier pseudospectral (TSGLFP2) method for the GPE (1.1) with $d=2$ as follows:

Let $\psi_{j s}^{0}=\psi_{0}\left(r_{j}, \theta_{s}\right)$ for $0 \leq j \leq K+M / 2$ and $0 \leq s \leq M-1$. For $n=0,1,2, \cdots$, we compute $\psi_{j s}^{n+1}(0 \leq j \leq K+M / 2,0 \leq s \leq M-1)$ by

$$
\begin{aligned}
& \psi_{j s}^{(1)}=e^{-i\left[W\left(r_{j}, \theta_{s}\right)+\beta_{2}\left|\psi_{j s}^{n}\right|^{2}\right] \Delta t / 2} \psi_{j s}^{n}, \\
& \psi_{j s}^{(2)}=\sum_{m=-M / 2}^{M / 2-1}\left[e^{i m \theta_{s}} \sum_{k=0}^{K} e^{-i \mu_{k m} \Delta t} \widehat{\psi^{(1)}} k m L_{k}^{|m|}\left(r_{j}\right)\right], \\
& \psi_{j s}^{n+1}=e^{-i\left[W\left(r_{j}, \theta_{s}\right)+\beta_{2}\left|\psi_{j s}^{(2)}\right|^{2}\right] \Delta t / 2} \psi_{j s}^{(2)},
\end{aligned}
$$

where $\left\{\widehat{\psi^{(1)}} k m\right\}$ are the expansion coefficients of $\psi^{(1)}$ given by $(2.33)$.

LEMMA 2.1. The TSGLFP2 method (2.35) is normalization conserving, i.e.

$$
\left\|\psi^{n}\right\|^{2} \equiv\left\|\psi^{0}\right\|^{2}, \quad \forall n \geq 1
$$

Proof. One derives immediately from (2.35) that $\left\|\psi^{(1)}\right\|^{2}=\left\|\psi^{n}\right\|^{2}$ and $\left\|\psi^{n+1}\right\|^{2}=$ $\left\|\psi^{(2)}\right\|^{2}$. By using (2.30), (2.32) and (2.33), we find

$$
\left\|\psi^{(2)}\right\|^{2}=\frac{2 \pi}{M} \sum_{s=0}^{M-1} \sum_{j=0}^{K+M / 2}\left|\psi_{j s}^{(2)}\right|^{2} \omega_{j}=\frac{2 \pi}{M} \sum_{s=0}^{M-1} \sum_{j=0}^{K+M / 2}\left|\psi_{j s}^{(1)}\right|^{2} \omega_{j}=\left\|\psi^{(1)}\right\|^{2} .
$$


Therefore, we have $\left\|\psi^{n+1}\right\|^{2}=\left\|\psi^{n}\right\|^{2}$ for all $n \geq 0$.

It is clear that the memory requirement of this scheme is $O((K+M / 2) M)$, and the computational cost per time step is $O(M(K+M / 2)(K+M / 2+\ln M))$.

2.3. Generalized-Laguerre-Fourier-Hermite pseudospectral method for rotating $\mathbf{B E C}$ in $3 \mathrm{D}$. In the $3 \mathrm{D}$ case, by using the cylindrical coordinates $(r, \theta, z)$, we can write the solutions of $(2.7)$ as $\psi(r, \theta, z, t)$. Therefore, for $t \geq t_{s}\left(t_{s}\right.$ is any given time), (2.7) collapses to

$$
\begin{aligned}
i \partial_{t} \psi(r, \theta, z, t) & =\frac{1}{2}\left[-\frac{1}{r} \frac{\partial}{\partial r}\left(r \frac{\partial}{\partial r}\right)-\frac{1}{r^{2}} \frac{\partial^{2}}{\partial \theta^{2}}-\frac{\partial^{2}}{\partial z^{2}}+\gamma_{r}^{2} r^{2}+\gamma_{z} z^{2}+2 i \Omega \partial_{\theta}\right] \psi, \\
& =\left(B_{\perp}+B_{z}\right) \psi(r, \theta, z, t)=B \psi(r, \theta, z, t), \\
\psi(r, \theta+2 \pi, z, t) & =\psi(r, \theta, z, t), \quad 0<r<\infty, \quad 0<\theta<2 \pi, \quad z \in \mathbb{R}, \\
\lim _{r \rightarrow \infty} \psi(r, \theta, z, t) & =0, \quad-\infty<z<\infty, \quad t \geq t_{s} .
\end{aligned}
$$

The normalization (1.4) collapses to

$$
\begin{aligned}
\|\psi(\cdot, t)\|^{2} & =\int_{-\infty}^{\infty} \int_{0}^{\infty} \int_{0}^{2 \pi}|\psi(r, \theta, z, t)|^{2} r d r d \theta d z \\
& =\int_{-\infty}^{\infty} \int_{0}^{\infty} \int_{0}^{2 \pi}\left|\psi_{0}(r, \theta, z)\right|^{2} r d r d \theta d z=1 .
\end{aligned}
$$

We now consider $B_{z}$ in $(2.2)$. To this end, let $H_{l}(z)(l=0,1,2, \ldots)$ be the standard Hermite polynomials of degree $l$ satisfying $[40,10,11,31,42]$

$$
\begin{aligned}
& H_{l}^{\prime \prime}(z)-2 z H_{l}^{\prime}(z)+2 l H_{l}(z)=0, \quad z \in \mathbb{R}, \quad l=0,1,2, \ldots, \\
& \int_{-\infty}^{\infty} H_{l}(z) H_{l^{\prime}}(z) e^{-z^{2}} d z=\sqrt{\pi} 2^{l} l ! \delta_{l l^{\prime}}, \quad l, l^{\prime}=0,1,2, \ldots
\end{aligned}
$$

As in $[10,11]$, we define the scaled Hermite functions

$$
h_{l}(z)=e^{-\gamma_{z} z^{2} / 2} H_{l}\left(\sqrt{\gamma_{z}} z\right) / \sqrt{2^{l} l !}\left(\gamma_{z} / \pi\right)^{1 / 4}, \quad z \in \mathbb{R} .
$$

It is clear that $\lim _{|z| \rightarrow \infty} h_{l}(z)=0$.

Plugging (2.41) into (2.39) and (2.40), a simple computation shows

$$
\begin{gathered}
B_{z} h_{l}(z)=-\frac{1}{2} h_{l}^{\prime \prime}(z)+\frac{1}{2} \gamma_{z}^{2} z^{2} h_{l}(z)=\lambda_{l} h_{l}(z), \quad z \in \mathbb{R}, \quad l \geq 0 \\
\int_{-\infty}^{\infty} h_{l}(z) h_{l^{\prime}}(z) d z=\delta_{l l^{\prime}}, \quad l, l^{\prime}=0,1,2, \ldots
\end{gathered}
$$

where

$$
\lambda_{l}=\left(l+\frac{1}{2}\right) \gamma_{z}, \quad l=0,1,2, \ldots .
$$

Hence $\left\{h_{l}\right\}_{l=0}^{\infty}$ are eigenfunctions of the linear operator $B_{z}$ in (2.2). 
Finally, for any fixed $m(m=0, \pm 1, \pm 2, \ldots)$, we derive from the above that

$$
\begin{aligned}
B\left(L_{k}^{|m|}(r) e^{i m \theta} h_{l}(z)\right) & =\left(B_{\perp}+B_{z}\right)\left(L_{k}^{|m|}(r) e^{i m \theta} h_{l}(z)\right) \\
& =h_{l}(z) B_{\perp}\left(L_{k}^{|m|}(r) e^{i m \theta}\right)+L_{k}^{|m|}(r) e^{i m \theta} B_{z} h_{l}(z) \\
& =\mu_{k m} L_{k}^{|m|}(r) e^{i m \theta} h_{l}(z)+\lambda_{l} L_{k}^{|m|}(r) e^{i m \theta} h_{l}(z) \\
& =\left(\mu_{k m}+\lambda_{l}\right) L_{k}^{|m|}(r) e^{i m \theta} h_{l}(z) .
\end{aligned}
$$

Hence, $\left\{L_{k}^{|m|}(r) e^{i m \theta} h_{l}(z), k, l=0,1, \cdots, m=0, \pm 1, \pm 2, \cdots\right\}$ are eigenfunctions of the linear operator $B=B_{\perp}+B_{z}$ defined in (2.3) for $d=3$.

For fixed even integer $M>0$ and integers $K>0$ and $L>0$, let

$Y_{K M L}=\operatorname{span}\left\{L_{k}^{|m|}(r) e^{i m \theta} h_{l}(z): 0 \leq k \leq K,-M / 2 \leq m \leq M / 2-1,0 \leq l \leq L\right\}$.

The generalized-Laguerre-Fourier-Hermite spectral method for (2.37) is:

find $\psi_{M K L}(r, \theta, z, t) \in Y_{K M L}$, i.e.

$$
\psi_{K M L}(r, \theta, z, t)=\sum_{l=0}^{L}\left[h_{l}(z) \sum_{m=-M / 2}^{M / 2-1}\left(e^{i m \theta} \sum_{k=0}^{K} \hat{\psi}_{k m l}(t) L_{k}^{|m|}(r)\right)\right]
$$

such that

$$
\begin{aligned}
i \frac{\partial \psi_{K M L}}{\partial t} & =\frac{1}{2}\left[-\frac{1}{r} \frac{\partial}{\partial r}\left(r \frac{\partial}{\partial r}\right)-\frac{1}{r^{2}} \frac{\partial^{2}}{\partial \theta^{2}}-\frac{\partial^{2}}{\partial z^{2}}+\gamma_{r}^{2} r^{2}+\gamma_{z}^{2} z^{2}+2 i \Omega \partial_{\theta}\right] \psi_{K M L}, \\
& =\left(B_{\perp}+B_{z}\right) \psi_{K M L}, \quad 0<r<\infty, \quad 0<\theta<2 \pi, \quad z \in \mathbb{R} .
\end{aligned}
$$

Plugging (2.46) into (2.47), thanks to (2.45), noticing the orthogonality of the Fourier series, we find

$$
i \frac{d \hat{\psi}_{k m l}(t)}{d t}=\left(\mu_{k m}+\lambda_{l}\right) \hat{\psi}_{k m l}(t), \quad t \geq t_{s}
$$

Similar as the 2D case, the above linear ODE can be integrated exactly, we obtain the solution of $(2.47)$ as

$$
\begin{aligned}
\psi_{K M L}(r, \theta, z, t)=e^{-i\left(t-t_{s}\right)\left(B_{\perp}+B_{z}\right)} \psi_{K M L}\left(r, \theta, z, t_{s}\right) \\
=\sum_{l=0}^{L}\left[h_{l}(z) \sum_{m=-M / 2}^{M / 2-1}\left(e^{i m \theta} \sum_{k=0}^{K} e^{-i\left(\mu_{k m}+\lambda_{l}\right)\left(t-t_{s}\right)} \hat{\psi}_{k m l}\left(t_{s}\right) L_{k}^{|m|}(r)\right)\right],
\end{aligned}
$$

where

$$
\hat{\psi}_{k m l}\left(t_{s}\right)=\frac{1}{2 \pi} \int_{-\infty}^{\infty}\left[h_{l}(z) \int_{0}^{2 \pi}\left(e^{-i m \theta} \int_{0}^{\infty} \psi_{K M L}\left(r, \theta, z, t_{s}\right) L_{k}^{|m|}(r) r d r\right) d \theta\right] d z .
$$

As in the $2 \mathrm{D}$ case, we need to approximate the above integral by a suitable quadrature rule. Let $\left\{\hat{z}_{p}\right\}_{p=0}^{L}$ be the Hermite-Gauss points, i.e., they are the $L+1$ roots of the Hermite polynomial $H_{L+1}(z)$, and let $\left\{\hat{\omega}_{p}^{z}\right\}_{p=0}^{L}$ be the associated HermiteGauss quadrature weights [40]. Then, we have

$$
\int_{-\infty}^{\infty} f(z) e^{-z^{2}} d z=\sum_{p=0}^{L} f\left(\hat{z}_{p}\right) \hat{\omega}_{p}^{z}, \quad \forall f \in P_{2 L+1},
$$


which implies that

$$
\begin{aligned}
\sum_{p=0}^{L} \hat{\omega}_{p}^{z} \frac{H_{l}\left(\hat{z}_{p}\right)}{\pi^{1 / 4} \sqrt{2^{l} l !}} \frac{H_{l^{\prime}}\left(\hat{z}_{p}\right)}{\pi^{1 / 4} \sqrt{2^{l^{\prime}} l^{\prime !}}} & =\int_{-\infty}^{\infty} \frac{H_{l}\left(\hat{z}_{p}\right)}{\pi^{1 / 4} \sqrt{2^{l} l !}} \frac{H_{l^{\prime}}\left(\hat{z}_{p}\right)}{\pi^{1 / 4} \sqrt{2^{l^{\prime}} l^{\prime !}}} e^{-z^{2}} d z \\
& =\delta_{l l^{\prime}}, \quad 0 \leq l, l^{\prime} \leq L .
\end{aligned}
$$

We then define the scaled Hermite-Gauss points and weights by

$$
z_{p}=\frac{\hat{z}_{p}}{\sqrt{\gamma_{z}}}, \quad \omega_{p}^{z}=\frac{\hat{\omega}_{p}^{z} e^{\hat{z}_{p}^{2}}}{\sqrt{\gamma_{z}}}, \quad p=0,1,2, \ldots, L
$$

We derive from (2.41) and (2.52) that

$$
\begin{aligned}
\sum_{p=0}^{L} \omega_{p}^{z} h_{l}\left(z_{p}\right) h_{l^{\prime}}\left(z_{p}\right) & =\sum_{p=0}^{L} \frac{\hat{\omega}_{p}^{z} e^{\hat{z}_{p}^{2}}}{\sqrt{\gamma_{z}}} h_{l}\left(\hat{z}_{p} / \sqrt{\gamma_{z}}\right) h_{l^{\prime}}\left(\hat{z}_{p} / \sqrt{\gamma_{z}}\right) \\
& =\sum_{p=0}^{L} \hat{\omega}_{p}^{z} \frac{H_{l}\left(\hat{z}_{p}\right)}{\pi^{1 / 4 \sqrt{2^{l} l !}}} \frac{H_{l^{\prime}}\left(\hat{z}_{p}\right)}{\pi^{1 / 4 \sqrt{2^{l^{\prime}} l^{\prime} !}}} \\
& =\delta_{l l^{\prime}}, \quad l, l^{\prime}=0,1, \ldots, L .
\end{aligned}
$$

Similarly, care must be taken when computing the weights $\left\{\omega_{p}^{z}\right\}$ for large $L$ (cf. the Appendix of [39]).

For any given set of values $\left\{\psi_{j s p}, 0 \leq j \leq K+M / 2 ; 0 \leq s \leq M-1 ; 0 \leq p \leq L\right\}$, we can define a unique function $\psi$ in $Y_{K M L}$ interpolating this set, i.e.,

$$
\begin{aligned}
& \psi(r, \theta, z)=\sum_{m=-M / 2}^{M / 2-1} \sum_{k=0}^{K} \sum_{l=0}^{L} \widehat{\psi}_{k m l} L_{k}^{|m|}(r) e^{i m \theta} h_{l}(z) \quad \text { such that } \\
& \psi\left(r_{j}, \theta_{s}, z_{p}\right)=\psi_{j s p}, \quad 0 \leq j \leq K+M / 2 ; 0 \leq s \leq M-1 ; 0 \leq p \leq L .
\end{aligned}
$$

By using the discrete orthogonality relations (2.30), (2.32) and (2.54), we find that

$$
\widehat{\psi}_{k m l}=\frac{1}{M} \sum_{p=0}^{L}\left[h_{l}\left(z_{p}\right) \omega_{p}^{z} \sum_{s=0}^{M-1}\left(e^{-i m \theta_{s}} \sum_{j=0}^{K+M / 2} \omega_{j} \psi_{j s p} L_{k}^{|m|}\left(r_{j}\right)\right)\right]
$$

and that

$$
\begin{aligned}
\|\psi\|^{2} & :=\int_{-\infty}^{\infty} \int_{0}^{\infty} \int_{0}^{2 \pi}|\psi(r, \theta, z)|^{2} r d \theta d r d z \\
& =2 \pi \sum_{m=-M / 2}^{M / 2-1} \sum_{k=0}^{K} \sum_{l=0}^{L}\left|\widehat{\psi}_{k m l}\right|^{2}=\frac{2 \pi}{M} \sum_{j=0}^{K+M / 2} \sum_{s=0}^{M-1} \sum_{p=0}^{L}\left|\psi_{j s p}\right|^{2} \omega_{j} \omega_{p}^{z} .
\end{aligned}
$$

Then the second-order time-splitting generalized-Laguerre-Fourier-Hermite pseudospectral (TSGLFHP2) method for the GPE (1.1) with $d=3$ is as follows: 
Let $\psi_{j s p}^{0}=\psi_{0}\left(r_{j}, \theta_{s}, z_{p}\right)$ for $0 \leq j \leq K+M / 2,0 \leq s \leq M-1$ and $0 \leq p \leq L$. For $n=0,1, \cdots$, we compute $\psi_{j s p}^{n+1}$ by

$$
\begin{aligned}
& \psi_{j s p}^{(1)}=e^{-i\left[W\left(r_{j}, \theta_{s}, z_{p}\right)+\beta_{3}\left|\psi_{j s p}^{n}\right|^{2}\right] \Delta t / 2} \psi_{j s p}^{n}, \\
& \psi_{j s p}^{(2)}=\sum_{l=0}^{L}\left[h_{l}\left(z_{p}\right) \sum_{m=-M / 2}^{M / 2-1}\left(e^{i m \theta_{s}} \sum_{k=0}^{K} e^{-i\left(\mu_{k m}+\lambda_{l}\right) \Delta t} \widehat{\psi^{(1)}}{ }_{k m l} L_{k}^{|m|}\left(r_{j}\right)\right)\right], \\
& \psi_{j s p}^{n+1}=e^{-i\left[W\left(r_{j}, \theta_{s}, z_{p}\right)+\beta_{3}\left|\psi_{j s p}^{(2)}\right|^{2}\right] \Delta t / 2} \psi_{j s p}^{(2)},
\end{aligned}
$$

where $\left\{\widehat{\psi^{(1)}}{ }_{k m l}\right\}$ are the expansion coefficients of $\psi^{(1)}$ given by $(2.56)$.

Following a similar procedure as in the proof of Lemma 2.1, we can prove the following:

LEMMA 2.2. The TSGLFHP2 method (2.58) is normalization conserving, i.e.

$$
\left\|\psi^{n}\right\|^{2}=\left\|\psi^{0}\right\|^{2}, \quad \forall n \geq 1
$$

The memory requirement of this scheme is $O((K+M / 2) M L)$, and the computational cost per time step is $O(M L(K+M / 2)(L+K+M / 2+\ln M))$.

3. Extension to rotating two-component and spin-1 BEC. The numerical methods TSGLFP2 for 2D, and resp. TSGLFHP2 for 3D, introduced above for GPE with an angular momentum rotation term, can be extended to coupled GrossPitaevskii equations (CGPEs) for the dynamics of rotating two-component and spin-1 BEC.

3.1. For rotating two-component BEC. Consider the dimensionless CGPEs with an angular momentum rotation term and an external driving field for the dynamics of rotating two-component BEC in $d$-dimensions $(d=2,3)[36,46,4]$

$$
\begin{aligned}
& i \partial_{t} \psi_{1}(\mathbf{x}, t)=\left[-\frac{1}{2} \Delta+V_{1}(\mathbf{x})-\Omega L_{z}+\beta_{11}\left|\psi_{1}\right|^{2}+\beta_{12}\left|\psi_{2}\right|^{2}\right] \psi_{1}-\lambda \psi_{2}, \\
& i \partial_{t} \psi_{2}(\mathbf{x}, t)=\left[-\frac{\alpha}{2} \Delta+V_{2}(\mathbf{x})-\Omega L_{z}+\beta_{21}\left|\psi_{1}\right|^{2}+\beta_{22}\left|\psi_{2}\right|^{2}\right] \psi_{2}-\lambda \psi_{1}, \\
& \psi_{1}(\mathbf{x}, 0)=\psi_{1}^{(0)}(\mathbf{x}), \quad \psi_{2}(\mathbf{x}, 0)=\psi_{2}^{(0)}(\mathbf{x}), \quad \mathbf{x} \in \mathbb{R}^{d} .
\end{aligned}
$$

Here, $\Psi=\Psi(\mathbf{x}, t):=\left(\psi_{1}(\mathbf{x}, t), \psi_{2}(\mathbf{x}, t)\right)^{T}$ is the dimensionless wave function of the rotating two-component BEC, $V_{1}(\mathbf{x})$ and $V_{2}(\mathbf{x})$ are the dimensionless external trapping potentials, $\alpha=\frac{m_{1}}{m_{2}}>0$ with $m_{1}$ and $m_{2}$ the atomic masses of the two species, $\Omega$ is the dimensionless angular velocity of the rotating laser beam, $\lambda$ is the dimensionless effective Rabi frequency describing the strength of the external driving field, and $\beta_{j l}=\frac{2 \pi N a_{j l}\left(m_{j}+m_{l}\right) m_{1}}{a_{0} m_{j} m_{l}}(j, l=1,2)$ with $N$ the total number of particles in the twocomponent condensate, $a_{j l}=a_{l j}(j, l=1,2)$ the $s$-wave scattering length between the $j$ th and $l$ th component and $a_{0}=\sqrt{\hbar / \omega m_{1}}$ is the dimensionless length unit. Again, we split the external trapping potentials into two parts:

$$
V_{1}(\mathbf{x})=V_{s}(\mathbf{x})+W_{1}(\mathbf{x}), \quad V_{2}(\mathbf{x})=\alpha V_{s}(\mathbf{x})+W_{2}(\mathbf{x}), \quad \mathbf{x} \in \mathbb{R}^{d},
$$

where $V_{s}(\mathbf{x})$ defined in (1.3) is the radial and cylindrical symmetric part when $d=2$ and $d=3$, respectively; and $W_{1}(\mathbf{x})$ and $W_{2}(\mathbf{x})$ are the rest parts of the external trapping potentials. 
Two important invariants of (3.1) are the normalization of the wave function $[46,4]$

$$
\begin{aligned}
N(\Psi) & =\int_{\mathbb{R}^{d}}\|\Psi(\mathbf{x}, t)\|^{2} d \mathbf{x}=\int_{\mathbb{R}^{d}} \sum_{j=1}^{2}\left|\psi_{j}(\mathbf{x}, t)\right|^{2} d \mathbf{x} \\
& \equiv \int_{\mathbb{R}^{d}} \sum_{j=1}^{2}\left|\psi_{j}^{(0)}(\mathbf{x})\right|^{2} d \mathbf{x}=N\left(\Psi^{(0)}\right)=1, \quad t \geq 0
\end{aligned}
$$

with $\Psi^{(0)}=\left(\psi_{1}^{(0)}, \psi_{2}^{(0)}\right)^{T}$, and the energy per particle

$$
\begin{aligned}
E_{\beta, \Omega}(\Psi)= & \int_{\mathbb{R}^{d}}\left[\sum_{j=1}^{2}\left(V_{j}(\mathbf{x})\left|\psi_{j}\right|^{2}-\Omega \operatorname{Re}\left(\bar{\psi}_{j} L_{z} \psi_{j}\right)+\sum_{l=1}^{2} \frac{\beta_{j l}}{2}\left|\psi_{j}\right|^{2}\left|\psi_{l}\right|^{2}\right)\right) \\
& \left.+\frac{1}{2}\left|\nabla \psi_{1}\right|^{2}+\frac{\alpha}{2}\left|\nabla \psi_{2}\right|^{2}-2 \lambda \operatorname{Re}\left(\bar{\psi}_{1} \psi_{2}\right)\right] d \mathbf{x} \equiv E_{\beta, \Omega}\left(\Psi^{(0)}\right), \quad t \geq 0 .
\end{aligned}
$$

In addition, if there is no external driving field, i.e. $\lambda=0$ in (3.1), the density of each component is also conserved, i.e.

$$
N_{j}(t):=N\left(\psi_{j}\right)=\int_{\mathbb{R}^{d}}\left|\psi_{j}(\mathbf{x}, t)\right|^{2} d \mathbf{x} \equiv\left\|\psi_{j}^{(0)}\right\|^{2}, \quad t \geq 0, \quad j=1,2 .
$$

Similar as for the case of single-component BEC, for $n=0,1,2, \ldots$, from time $t=t_{n}=n \Delta t$ to $t=t_{n+1}=t_{n}+\Delta t$, the CGPEs (3.1) can be solved in two splitting steps. One first solves

$$
\begin{aligned}
& i \partial_{t} \psi_{1}(\mathbf{x}, t)=\left[-\frac{1}{2} \Delta+V_{s}(\mathbf{x})-\Omega L_{z}\right] \psi_{1}-\lambda \psi_{2}=B \psi_{1}-\lambda \psi_{2} \\
& i \partial_{t} \psi_{2}(\mathbf{x}, t)=\left[\alpha\left(-\frac{1}{2} \Delta+V_{s}(\mathbf{x})\right)-\Omega L_{z}\right] \psi_{2}-\lambda \psi_{1}=\tilde{B} \psi_{2}-\lambda \psi_{1}, \quad \mathbf{x} \in \mathbb{R}^{d},
\end{aligned}
$$

for the time step of length $\Delta t$, followed by solving

$$
\begin{aligned}
& i \partial_{t} \psi_{1}(\mathbf{x}, t)=\left[W_{1}(\mathbf{x})+\beta_{11}\left|\psi_{1}\right|^{2}+\beta_{12}\left|\psi_{2}\right|^{2}\right] \psi_{1}, \\
& i \partial_{t} \psi_{2}(\mathbf{x}, t)=\left[W_{2}(\mathbf{x})+\beta_{21}\left|\psi_{1}\right|^{2}+\beta_{22}\left|\psi_{2}\right|^{2}\right] \psi_{2}, \quad \mathbf{x} \in \mathbb{R}^{d},
\end{aligned}
$$

for the same time step. For $t \in\left[t_{n}, t_{n+1}\right]$, the nonlinear ODE system (3.7) leaves $\left|\psi_{1}(\mathbf{x}, t)\right|$ and $\left|\psi_{2}(\mathbf{x}, t)\right|$ invariant, and thus can be integrated exactly. For simplicity, we present below only the extension for the scheme TSGLFP2 in 2D with $\alpha=1$. The others can be done in a similar way and we omit the details here for brevity.

The second-order time-splitting generalized-Laguerre-Fourier pseudospectral (TSGLFP2) method for the CGPEs (3.1) with $d=2$ is as follows:

Let $\left(\psi_{1}^{0}\right)_{j s}=\psi_{1}^{(0)}\left(r_{j}, \theta_{s}\right)$ and $\left(\psi_{2}^{0}\right)_{j s}=\psi_{2}^{(0)}\left(r_{j}, \theta_{s}\right)$ for $0 \leq j \leq K+M / 2$ and 
$0 \leq s \leq M-1$. For $n=0,1,2, \cdots$, we compute $\left(\psi_{1}^{n+1}\right)_{j s}$ and $\left(\psi_{2}^{n+1}\right)_{j s}$ by

$$
\begin{aligned}
& \left(\psi_{1}^{(1)}\right)_{j s}=e^{-i\left[W_{1}\left(r_{j}, \theta_{s}\right)+\beta_{11}\left|\left(\psi_{1}^{n}\right)_{j s}\right|^{2}+\beta_{12}\left|\left(\psi_{2}^{n}\right)_{j s}\right|^{2}\right] \Delta t / 2}\left(\psi_{1}^{n}\right)_{j s}, \\
& \left(\psi_{2}^{(1)}\right)_{j s}=e^{-i\left[W_{2}\left(r_{j}, \theta_{s}\right)+\beta_{21}\left|\left(\psi_{1}^{n}\right)_{j s}\right|^{2}+\beta_{22}\left|\left(\psi_{2}^{n}\right)_{j s}\right|^{2}\right] \Delta t / 2}\left(\psi_{2}^{n}\right)_{j s}, \\
& \left(\psi_{1}^{(2)}\right)_{j s}=\sum_{m=-M / 2}^{M / 2-1}\left[e^{i m \theta_{s}} \sum_{k=0}^{K} e^{-i \mu_{k m} \Delta t}\left[\cos (\lambda \Delta t) \widehat{\left(\psi_{1}^{(1)}\right)_{k m}}+i \sin (\lambda \Delta t)\left(\widehat{\psi_{2}^{(1)}}\right)_{k m}\right] L_{k}^{|m|}\left(r_{j}\right)\right] \text {, } \\
& \left.\left(\psi_{2}^{(2)}\right)_{j s}=\sum_{m=-M / 2}^{M / 2-1}\left[e^{i m \theta_{s}} \sum_{k=0}^{K} e^{-i \mu_{k m} \Delta t}\left[i \sin (\lambda \Delta t) \widehat{\left(\psi_{1}^{(1)}\right.}\right)_{k m}+\cos (\lambda \Delta t) \widehat{\left(\psi_{2}^{(1)}\right)_{k m}}\right] L_{k}^{|m|}\left(r_{j}\right)\right] \text {, } \\
& \left(\psi_{1}^{n+1}\right)_{j s}=e^{-i\left[W_{1}\left(r_{j}, \theta_{s}\right)+\beta_{11}\left|\left(\psi_{1}^{(2)}\right)_{j s}\right|^{2}+\beta_{12}\left|\left(\psi_{2}^{(2)}\right)_{j s}\right|^{2}\right] \Delta t / 2}\left(\psi_{1}^{(2)}\right)_{j s}, \\
& \left(\psi_{2}^{n+1}\right)_{j s}=e^{-i\left[W_{2}\left(r_{j}, \theta_{s}\right)+\beta_{21}\left|\left(\psi_{1}^{(2)}\right)_{j s}\right|^{2}+\beta_{22}\left|\left(\psi_{2}^{(2)}\right)_{j s}\right|^{2}\right] \Delta t / 2}\left(\psi_{2}^{(2)}\right)_{j s} ;
\end{aligned}
$$

where $\left(\widehat{\psi_{1}^{(1)}}\right)_{k m}$ and $\left(\widehat{\psi_{2}^{(1)}}\right)_{k m}$ are the generalized-Laguerre-Fourier transform coefficients of $\psi_{1}^{(1)}$ and $\psi_{2}^{(1)}$ given by (2.33).

Using the same argument as in the proof of Lemma 2.1, we can prove the following:

LEMMA 3.1. The above TSGLFP2 method for rotating two-component BEC is normalization conserving, i.e.

$$
\left\|\Psi^{n}\right\|^{2}:=\int_{0}^{\infty} \int_{0}^{2 \pi} \sum_{j=1}^{2}\left|\psi_{j}^{n}(r, \theta)\right|^{2} r d \theta d r \equiv\left\|\Psi^{0}\right\|^{2}, \quad n \geq 1 .
$$

In addition, if there is no external driving field, i.e. $\lambda=0$ in (3.1), the density of each component is also conserved, i.e.

$$
\left\|\psi_{j}^{n}\right\|^{2}:=\int_{0}^{\infty} \int_{0}^{2 \pi}\left|\psi_{j}^{n}(r, \theta)\right|^{2} r d \theta d r \equiv\left\|\psi_{j}^{0}\right\|^{2}, \quad n \geq 1, \quad j=1,2 .
$$

3.2. For rotating spin-1 BEC. Consider the dimensionless CGPEs with an angular momentum rotation term for the dynamics of rotating spin-1 BEC in $d$ dimensions $(d=2,3)[23,8]$

$$
\begin{aligned}
& i \partial_{t} \psi_{1}(\mathbf{x}, t)=\left[H+W_{1}(\mathbf{x})+\beta_{n} \rho+\beta_{s}\left(\rho_{1}+\rho_{0}-\rho_{-1}\right)\right] \psi_{1}+\beta_{s} \bar{\psi}_{-1} \psi_{0}^{2}, \\
& i \partial_{t} \psi_{0}(\mathbf{x}, t)=\left[H+W_{0}(\mathbf{x})+\beta_{n} \rho+\beta_{s}\left(\rho_{1}+\rho_{-1}\right)\right] \psi_{0}+2 \beta_{s} \psi_{-1} \bar{\psi}_{0} \psi_{1}, \\
& i \partial_{t} \psi_{-1}(\mathbf{x}, t)=\left[H+W_{-1}(\mathbf{x})+\beta_{n} \rho+\beta_{s}\left(\rho_{-1}+\rho_{0}-\rho_{1}\right)\right] \psi_{-1}+\beta_{s} \psi_{0}^{2} \bar{\psi}_{1}, \\
& \psi_{j}(\mathbf{x}, 0)=\psi_{j}^{(0)}(\mathbf{x}), \quad \mathbf{x} \in \mathbb{R}^{d}, \quad j=-1,0,1 .
\end{aligned}
$$

Here, $\Psi=\Psi(\mathbf{x}, t):=\left(\psi_{1}(\mathbf{x}, t), \psi_{0}(\mathbf{x}, t), \psi_{-1}(\mathbf{x}, t)\right)^{T}$ is the dimensionless wave function of the rotating spin-1 BEC, $H=-\frac{1}{2} \Delta+V_{s}(\mathbf{x})-\Omega L_{z}$ with $V_{s}(\mathbf{x})$ defined in (1.3), $\Omega$ is the dimensionless angular velocity of the rotating laser beam, $W_{j}(\mathbf{x})(j=-1,0,1)$ are the rest of the external trapping potentials, $\rho_{j}=\rho_{j}(\mathbf{x}, t):=\left|\psi_{j}(\mathbf{x}, t)\right|^{2}$ is the spatial density of the hyperfine spin component $m_{F}=j(j=-1,0,1)$ and $\rho=\rho_{1}+\rho_{0}+\rho_{-1}$ is the total density. $\beta_{n}=\frac{4 \pi N\left(a_{0}+2 a_{2}\right)}{a_{s}}$ and $\beta_{s}=\frac{4 \pi N\left(a_{2}-a_{0}\right)}{a_{s}}$ are the dimensionless mean-field and spin-exchange interaction constants, respectively, with $N$ the total number of particles in the spin- 1 condensate, $a_{0}$ and $a_{2}$ the $s$-wave scattering length 
for scattering channel of total hyperfine spin 0 and 2 , respectively, and $a_{s}=\sqrt{\hbar / \omega m_{b}}$ the dimensionless length unit.

Three important invariants of (3.10) are the normalization of the wave function $[23,8]$

$$
\begin{aligned}
N(\Psi) & =\int_{\mathbb{R}^{d}}\|\Psi(\mathbf{x}, t)\|^{2} d \mathbf{x}=\int_{\mathbb{R}^{d}} \sum_{j=-1}^{1}\left|\psi_{j}(\mathbf{x}, t)\right|^{2} d \mathbf{x} \\
& \equiv \int_{\mathbb{R}^{d}} \sum_{j=-1}^{1}\left|\psi_{j}^{(0)}(\mathbf{x})\right|^{2} d \mathbf{x}=N\left(\Psi^{(0)}\right)=1, \quad t \geq 0,
\end{aligned}
$$

with $\Psi^{(0)}=\left(\psi_{1}^{(0)}, \psi_{0}^{(0)}, \psi_{-1}^{(0)}\right)^{T}$, the total magnetization

$$
\begin{aligned}
M(\Psi) & =\int_{\mathbb{R}^{d}}\left[\left|\psi_{1}(\mathbf{x}, t)\right|^{2}-\left|\psi_{-1}(\mathbf{x}, t)\right|^{2}\right] d \mathbf{x} \\
& \equiv \int_{\mathbb{R}^{d}}\left[\left|\psi_{1}^{(0)}(\mathbf{x})\right|^{2}-\left|\psi_{-1}^{(0)}(\mathbf{x})\right|^{2}\right] d \mathbf{x}=M, \quad t \geq 0,
\end{aligned}
$$

with $-1 \leq M \leq 1$, and the energy per particle

$$
\begin{aligned}
E_{\beta, \Omega}(\Psi)= & \int_{\mathbb{R}^{d}}\left[\sum_{j=-1}^{1}\left(\frac{1}{2}\left|\nabla \psi_{j}\right|^{2}+\left(V_{s}(\mathbf{x})+W_{j}(\mathbf{x})\right)\left|\psi_{j}\right|^{2}-\Omega \operatorname{Re}\left(\bar{\psi}_{j} L_{z} \psi_{j}\right)\right)\right. \\
& +\frac{\beta_{n}}{2}\left|\rho_{0}\right|^{2}+\frac{\beta_{n}+\beta_{s}}{2}\left(\rho_{1}^{2}+\rho_{-1}^{2}+2 \rho_{0}\left(\rho_{1}+\rho_{-1}\right)\right)+\left(\beta_{n}-\beta_{s}\right) \rho_{1} \rho_{-1} \\
& \left.+\beta_{s}\left(\bar{\psi}_{1} \psi_{0}^{2} \bar{\psi}_{1}+\psi_{-1} \bar{\psi}_{0}^{2} \psi_{1}\right)\right] d \mathbf{x} \\
\equiv & E_{\beta, \Omega}\left(\Psi^{(0)}\right), \quad t \geq 0 .
\end{aligned}
$$

Unlike the TSGLFP2 method for GPE (1.1) and CGPEs (3.1), here it is advantageous to split the CGPEs (3.10) into three subsystems. More precisely, we rewrite (3.10) as

$$
i \partial_{t} \Psi(\mathbf{x}, t)=A \Psi+B \Psi+\beta_{s} C \Psi
$$

where

$$
\begin{gathered}
A=\operatorname{diag}\{H, H, H\}, \quad B=\operatorname{diag}\left\{b_{1}, b_{2}, b_{3}\right\}, \\
C=C(\Psi)=\left(\begin{array}{ccc}
0 & \bar{\psi}_{-1} \psi_{0} & 0 \\
\psi_{-1} \bar{\psi}_{0} & 0 & \bar{\psi}_{0} \psi_{1} \\
0 & \psi_{0} \bar{\psi}_{1} & 0
\end{array}\right) ;
\end{gathered}
$$

with

$$
\begin{aligned}
& H=-\frac{1}{2} \Delta+V_{s}(\mathbf{x})-\Omega L_{z}, \quad b_{1}=W_{1}(\mathbf{x})+\beta_{n} \rho+\beta_{s}\left(\rho_{1}+\rho_{0}-\rho_{-1}\right), \\
& \left.b_{2}=W_{0}(\mathbf{x})+\beta_{n} \rho+\beta_{s}\left(\rho_{1}+\rho_{-1}\right), \quad b_{3}=W_{-1}(\mathbf{x})+\beta_{n} \rho+\beta_{s}\left(\rho_{-1}+\rho_{0}-\rho_{1}\right)\right) .
\end{aligned}
$$

As shown in the previous section, the first subsystem $i \partial_{t} \Psi(\mathbf{x}, t)=A \Psi$ can be discretized in space by the generalized-Laguerre-Fourier method in $2 \mathrm{D}$, and resp, the 
generalized-Laguerre-Fourier-Hermite method in 3D, and integrated in time exactly. The second subsystem $i \partial_{t} \Psi(\mathbf{x}, t)=B \Psi$ is a nonlinear ODE system that leaves $\left|\psi_{1}(\mathbf{x}, t)\right|,\left|\psi_{0}(\mathbf{x}, t)\right|$ and $\left|\psi_{-1}(\mathbf{x}, t)\right|$ invariant in time $t$, and thus can be integrated exactly. The third subsystem $i \partial_{t} \Psi(\mathbf{x}, t)=\beta_{s} C(\Psi) \Psi$ is a nonlinear ODE system which can not be solved exactly. We shall take the approach used in [9], namely integrating it over the time integral $\left[t_{n}, t_{n+1}\right]$ and then approximating the integral by the second-order Runge-Kutta approximation [9],

$$
\begin{aligned}
\Psi^{n+1} & \approx \Psi\left(t_{n+1}\right)=e^{-i \beta_{s} \int_{t_{n}}^{t_{n}+1} C(\Psi(\tau)) d \tau} \Psi\left(t_{n}\right) \\
& \approx e^{-i \Delta t \beta_{s}\left(C\left(\Psi^{n}\right)+C\left(\Psi^{(1)}\right)\right) / 2} \Psi^{n}:=e^{-i \Delta t \beta_{s} D\left(\Psi^{n}\right)} \Psi^{n},
\end{aligned}
$$

where

$$
\begin{aligned}
& \Psi^{(1)}=\Psi^{n}-i \Delta t \beta_{s} C\left(\Psi^{n}\right) \Psi^{n}:=\left(\psi_{1}^{(1)}, \psi_{0}^{(1)}, \psi_{-1}^{(1)}\right)^{T}, \\
& D\left(\Psi^{n}\right)=\frac{1}{2}\left(C\left(\Psi^{n}\right)+C\left(\Psi^{(1)}\right)\right):=\left(\begin{array}{ccc}
0 & d_{12} & 0 \\
\bar{d}_{12} & 0 & d_{23} \\
0 & \bar{d}_{23} & 0
\end{array}\right),
\end{aligned}
$$

with

$$
d_{12}=\frac{1}{2}\left(\bar{\psi}_{-1}^{n} \psi_{0}^{n}+\bar{\psi}_{-1}^{(1)} \psi_{0}^{(1)}\right), \quad d_{23}=\frac{1}{2}\left(\bar{\psi}_{0}^{n} \psi_{1}^{n}+\bar{\psi}_{0}^{(1)} \psi_{1}^{(1)}\right) .
$$

Since $C(\Psi)$ is a Hermitian matrix, thus $D\left(\Psi^{n}\right)$ is also a Hermitian matrix, following [9], we can explicitly compute the approximation in (3.17) as

$$
\Psi^{n+1}=e^{-i \Delta t \beta_{s} D\left(\Psi^{n}\right)} \Psi^{n}=P e^{-i \beta_{s} \Delta t \Lambda} \bar{P}^{T} \Psi^{n},
$$

where

$$
\Lambda=\left(\begin{array}{ccc}
0 & 0 & 0 \\
0 & \lambda & 0 \\
0 & 0 & -\lambda
\end{array}\right), \quad P=\frac{1}{\sqrt{2} \lambda}\left(\begin{array}{ccc}
\sqrt{2} d_{23} & d_{12} & -d_{12} \\
0 & \lambda & \lambda \\
-\sqrt{2} d_{12} & \bar{d}_{23} & -\bar{d}_{23}
\end{array}\right)
$$

with

$$
\lambda=\sqrt{\left|d_{12}\right|^{2}+\left|d_{23}\right|^{2}} .
$$

Therefore, we can use the second-order Strang splitting pseudospectal method (TSGLFP2) to solve (3.14). We omit the detailed algorithms here for brevity.

Using the same argument as in the proof of Lemma 2.1, noticing that $D\left(\Psi^{n}\right)$ is a Hermitian matrix, we can prove the following:

LEMMA 3.2. The above TSGLFP2 method for rotating spin-1 BEC is normalization conserving, i.e.

$$
\begin{aligned}
\left\|\Psi^{n}\right\|^{2} & :=\int_{0}^{\infty} \int_{0}^{2 \pi} \sum_{j=-1}^{1}\left|\psi_{j}^{n}(r, \theta)\right|^{2} r d \theta d r \\
& \equiv\left\|\Psi^{0}\right\|^{2}, \quad n \geq 1 .
\end{aligned}
$$

REMARK 3.1. Another way to discretize the third subproblem $i \partial_{t} \Psi(\mathbf{x}, t)=\beta_{s} C(\Psi) \Psi$ is as following

$$
\Psi^{n+1}=\left(I+i \beta_{s} \Delta t C\left(\Psi^{n}\right) / 2\right)^{-1}\left(I-i \beta_{s} \Delta t C\left(\Psi^{n}\right) / 2\right) \Psi^{n},
$$

where $I$ is the $3 \times 3$ identity matrix. It is easy to show that this discretization is normalization conserving and second-order in time, too. 
4. Numerical results. We now present some numerical results by using the numerical methods introduced in previous sections to compute the dynamics of rotating BEC. To quantify the numerical results of a solution $\psi(\mathbf{x}, t)$, we define the condensate widths along the $r$ - and $z$-axes as $\sigma_{r}$ and $\sigma_{z}$ by

$$
\sigma_{\alpha}^{2}=\int_{\mathbb{R}^{d}} \alpha^{2}|\psi(\mathbf{x}, t)|^{2} d \mathbf{x}, \quad \alpha=x, y, z ; \quad \sigma_{r}^{2}=\sigma_{x}^{2}+\sigma_{y}^{2},
$$

and the angular momentum expectation which is a measure of the vortex flux by

$$
\left\langle L_{z}\right\rangle(t):=\int_{\mathbb{R}^{d}} \bar{\psi}(\mathbf{x}, t) L_{z} \psi(\mathbf{x}, t) d \mathbf{x}=i \int_{\mathbb{R}^{d}} \bar{\psi}(\mathbf{x}, t)\left(y \partial_{x}-x \partial_{y}\right) \psi(\mathbf{x}, t) d \mathbf{x} .
$$

Example 1. Dynamics of a rotating BEC in $2 \mathrm{D}$, i.e. we take $d=2, \beta_{2}=100, \Omega=0.5$ and $W(x, y)=\left(\gamma_{y}^{2}-\gamma_{x}^{2}\right) y^{2} / 2$ in (1.1). The initial data in (1.1) is chosen as

$$
\psi_{0}(x, y)=\frac{x+i y}{\sqrt{\pi}} e^{-\left(x^{2}+y^{2}\right) / 2}, \quad(x, y) \in \mathbb{R}^{2} .
$$

We solve the problem by the scheme (2.35) with $\Delta t=0.0005, M=128$ and $K=$ 100. Figure 1 depicts time evolution of the normalization $N(\psi)$, energy $E_{\beta, \Omega}(\psi)$, condensate width $\delta_{r}(t)$ and angular momentum expectation $\left\langle L_{z}\right\rangle(t)$ for three sets of parameters in (1.1): (i) $\gamma_{x}=\gamma_{y}=2:=\gamma_{r}$, (ii) $\gamma_{x}=\gamma_{y}=0.8:=\gamma_{r}$, and (iii) $\gamma_{x}=0.8:=\gamma_{r}, \gamma_{y}=1.2$.

From Fig. 1, we can draw the following conclusions: (i) the normalization $N(\psi)$ and energy $E_{\beta, \Omega}(\psi)$ are conserved well in the computation (cf. Fig. 1a\&b); (ii) the angular momentum expectation $\left\langle L_{z}\right\rangle(t)$ is conserved when $\gamma_{x}=\gamma_{y}$ (cf. Fig. 1d), i.e. the trapping is radial symmetric, which again confirms the analytical results in [5]; (iii) the condensate width $\delta_{r}(t)$ is a periodic function when $\gamma_{x}=\gamma_{y}$ (cf. Fig. 1c), which again confirms the analytical results in [5].

Example 2. Dynamics of a rotating BEC in 3D, i.e. we take $d=3, \beta_{3}=\beta=100$, $\Omega=0.5$ and $W(x, y, z)=\left(\gamma_{y}^{2}-\gamma_{x}^{2}\right) y^{2} / 2$ in (1.1). The initial data in (1.1) is chosen as

$$
\psi_{0}(x, y, z)=\frac{x+i y}{\pi^{3 / 4}} e^{-\left(x^{2}+y^{2}+z^{2}\right) / 2}, \quad(x, y, z) \in \mathbb{R}^{3} .
$$

We solve the problem by the scheme (2.58) with $\Delta t=0.0005, M=128, K=100$ and $L=50$. Figure 2 depicts time evolution of the energy $E_{\beta, \Omega}(\psi)$, condensate widths $\delta_{r}(t)$ and $\delta_{z}(t)$, and angular momentum expectation $\left\langle L_{z}\right\rangle(t)$ for three sets of parameters in the (1.1): (i) $\gamma_{x}=\gamma_{y}=2:=\gamma_{r}, \gamma_{z}=0.8$, (ii) $\gamma_{x}=\gamma_{y}=0.8:=\gamma_{r}$, $\gamma_{z}=2$, and (iii) $\gamma_{x}=0.8:=\gamma_{r}, \gamma_{y}=1.2, \gamma_{z}=1.2$.

From Fig. 2 and additional results not shown here for brevity, we can draw the following conclusions: (i) the energy $E_{\beta, \Omega}(\psi)$ and normalization $N(\psi)$ are conserved well in the computation (cf. Fig. 2a); (ii) the angular momentum expectation $\left\langle L_{z}\right\rangle(t)$ is conserved when $\gamma_{x}=\gamma_{y}$ (cf. Fig. 2d), i.e. the trapping is cylindrical symmetric, which again confirms the analytical results in [5]; (iii) the condensate widths $\delta_{r}(t)$ and $\delta_{z}(t)$ are periodic functions with perturbations (cf. Fig. 2b\&c), which again confirms the analytical results in [5].

Example 3. Dynamics of a rotating two-component BEC in 2D, i.e. we take $d=2$, $\Omega=0.5, \gamma_{x}=\gamma_{y}=2:=\gamma_{r}, \alpha=1, W_{1}(\mathbf{x}) \equiv 2$ and $W_{2}(\mathbf{x}) \equiv 0$ in (3.1). The initial 

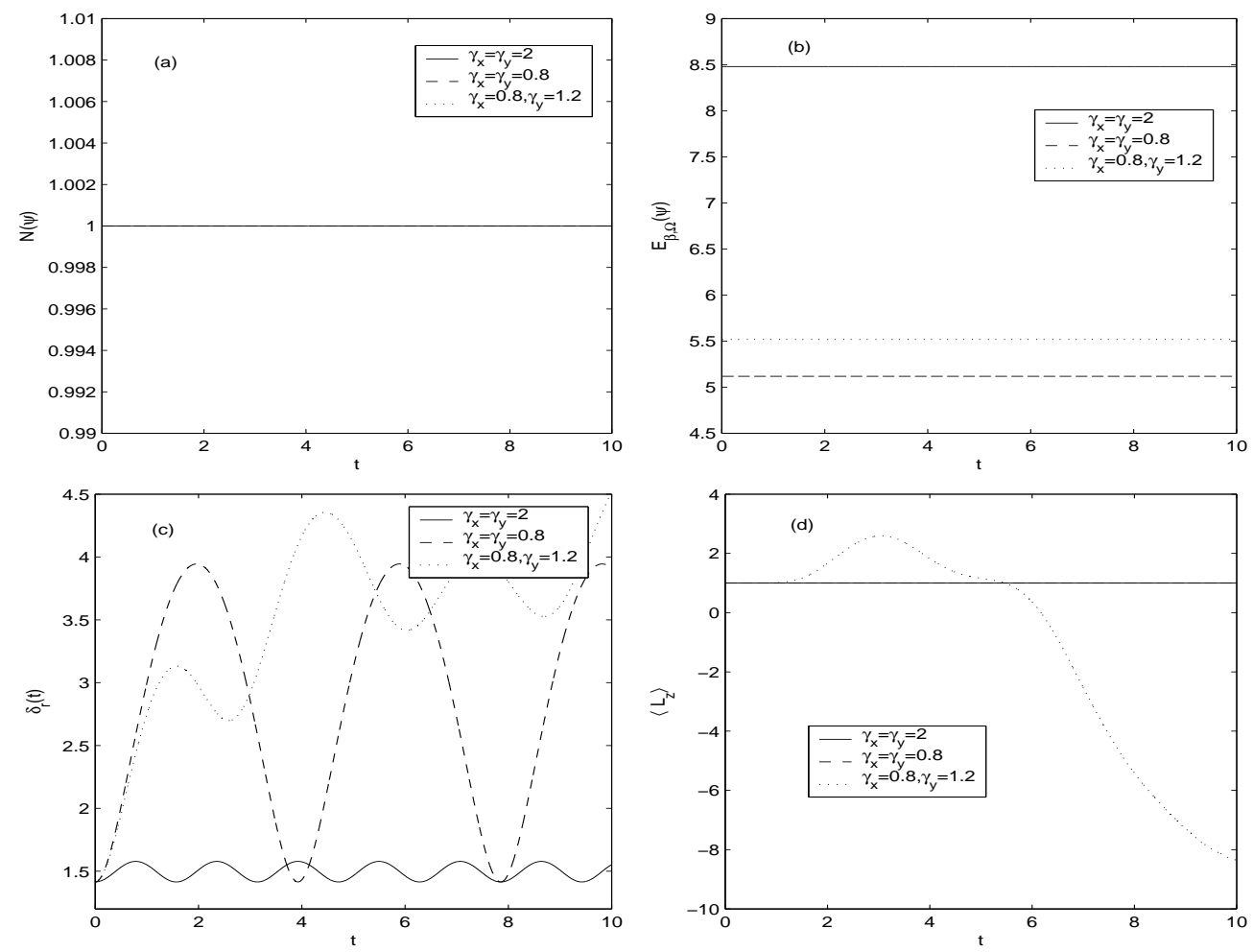

FIG. 1. Time evolution of a few quantities for the dynamics of rotating BEC in $2 D$ with three sets of parameters: (a) normalization $N(\psi)$, (b) energy $E_{\beta, \Omega}(\psi),(c)$ condensate width $\delta_{r}(t)$, and (d) angular momentum expectation $\left\langle L_{z}\right\rangle(t)$.

data in (3.1) is chosen as

$$
\psi_{1}^{(0)}(x, y)=\frac{4(x+i y)}{5 \sqrt{\pi}} e^{-\left(x^{2}+y^{2}\right) / 2}, \quad \psi_{2}^{(0)}(x, y)=\frac{3(x+i y)}{5 \sqrt{\pi}} e^{-\left(x^{2}+y^{2}\right) / 2} .
$$

We solve the problem by our numerical method with $\Delta t=0.0005, M=128$ and $K=100$. To additionally quantify the numerical results of a solution $\Psi(\mathbf{x}, t)=$ $\left(\psi_{1}(\mathbf{x}, t), \psi_{2}(\mathbf{x}, t)\right)^{T}$, we define [4]

$$
\begin{aligned}
& W_{1}(t)=i \int_{\mathbb{R}^{d}}\left[\bar{\psi}_{1}(\mathbf{x}, t) \psi_{2}(\mathbf{x}, t)-\psi_{1}(\mathbf{x}, t) \bar{\psi}_{2}(\mathbf{x}, t)\right] d \mathbf{x}, \\
& W_{2}(t)=i \int_{\mathbb{R}^{d}}\left[\bar{\psi}_{1}(\mathbf{x}, t) \psi_{2}(\mathbf{x}, t)+\psi_{1}(\mathbf{x}, t) \bar{\psi}_{2}(\mathbf{x}, t)\right] d \mathbf{x}, \quad t \geq 0 .
\end{aligned}
$$

Figure 3 depicts time evolution of the total density $N(t):=N(\Psi)$, density of each component $N_{j}(t)=N\left(\psi_{j}\right)(j=1,2)$, and $W_{1}(t)$ and $W_{2}(t)$ for four sets of parameters in (3.1): (i) $\beta_{11}=\beta_{12}=\beta_{22}=100, \lambda=0$, (ii) $\beta_{11}=100, \beta_{12}=80, \beta_{22}=120, \lambda=0$, (iii) $\beta_{11}=\beta_{12}=\beta_{22}=100, \lambda=\sqrt{3}$, (iv) $\beta_{11}=100, \beta_{12}=80, \beta_{22}=120, \lambda=\sqrt{3}$.

From Fig. 3 and our additional numerical results omitted here for brevity, we can draw the following conclusions: (i) the density of each component $N_{1}(t)$ and $N_{2}(t)$ are periodic functions when $\beta_{11}=\beta_{12}=\beta_{22}$ (cf. Fig. 3c), which confirms the analytical results in $[4,46]$; (ii) $W_{1}(t)$ and $W_{2}(t)$ are periodic functions when $\beta_{11}=\beta_{12}=\beta_{22}$ 

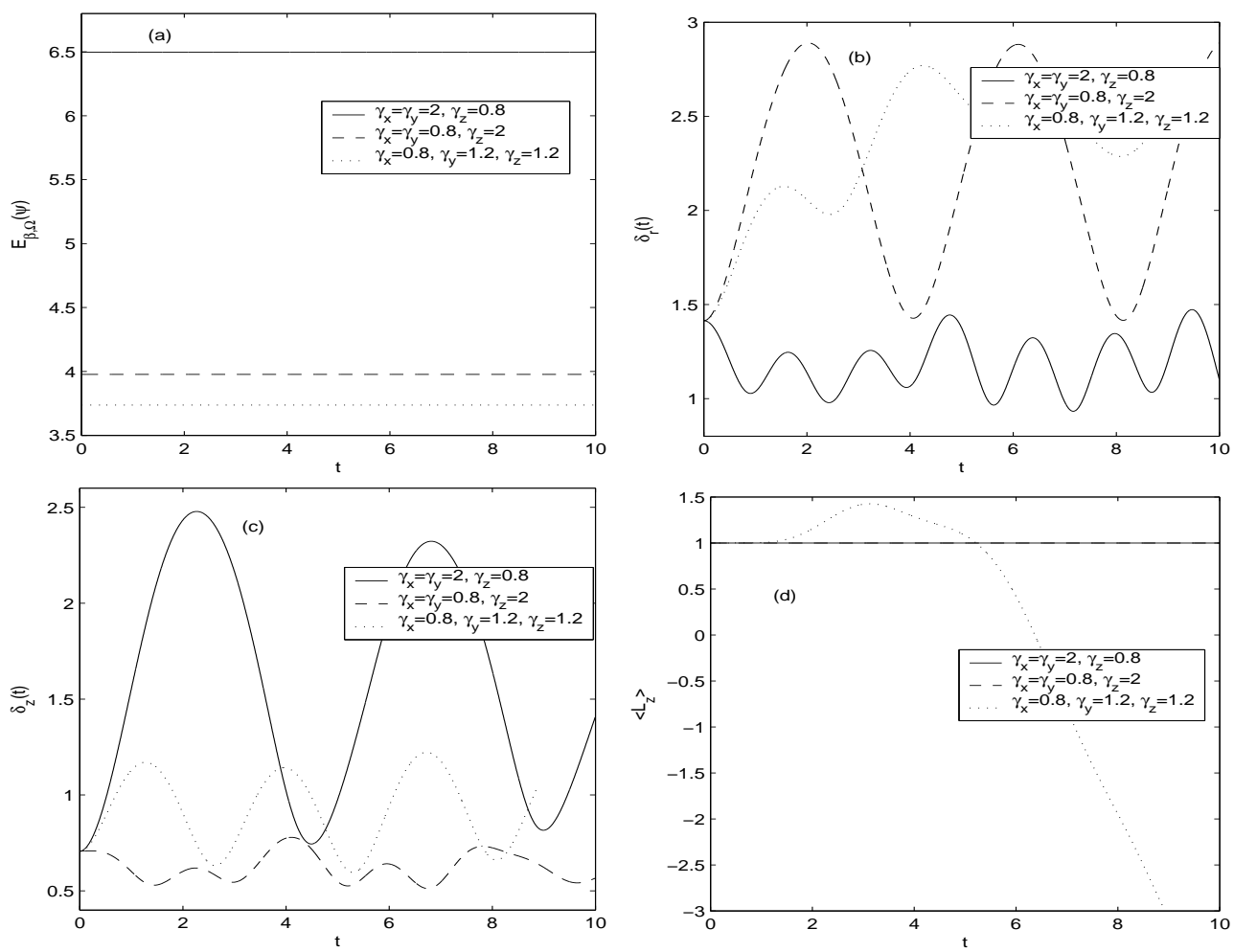

FIG. 2. Time evolution of a few quantities for the dynamics of rotating BEC in $3 D$ with three sets of parameters: (a) energy $E_{\beta, \Omega}(\psi),(b)$ condensate width $\delta_{r}(t),(c)$ condensate width $\delta_{z}(t)$, and (d) angular momentum expectation $\left\langle L_{z}\right\rangle(t)$.

(cf. Fig. 3a\&c), which confirms the analytical results in $[4,46]$; (iii) the normalization $N(\Psi)$ and energy $E_{\beta, \Omega}(\Psi)$ are conserved well in the computation; (iv) the angular momentum expectation $\left\langle L_{z}\right\rangle(t)$ is conserved when $\gamma_{x}=\gamma_{y}$, i.e. the trapping is radial symmetric, which again confirms the analytical results in $[4,46]$.

Example 4. Interaction of two vortex pairs and dipoles in a rotating BEC in 2D, i.e. we take $d=2, \beta_{2}=100, \Omega=0.5, \gamma_{x}=\gamma_{y}=1:=\gamma_{r}$ and $W(\mathbf{x}) \equiv 0$ in (1.1). The initial data in (1.1) is chosen as

Case I. Interaction of two vortex pairs, i.e.

$\psi_{0}(x, y)=C\left(\left(x-x_{0}\right)+i y\right)\left(\left(x+x_{0}\right)+i y\right)\left(x+i\left(y-y_{0}\right)\right)\left(x+i\left(y+y_{0}\right)\right) e^{-\left(x^{2}+y^{2}\right) / 2}$,

Case II. Interaction of two vortex dipoles, i.e.

$\psi_{0}(x, y)=C\left(\left(x-x_{0}\right)+i y\right)\left(\left(x+x_{0}\right)+i y\right)\left(x-i\left(y-y_{0}\right)\right)\left(x-i\left(y+y_{0}\right)\right) e^{-\left(x^{2}+y^{2}\right) / 2}$,

where the constant $C$ is chosen such that $\left\|\psi_{0}\right\|^{2}=\int_{\mathbb{R}^{2}}\left|\psi_{0}(x, y)\right|^{2} d x d y=1$ and we take $x_{0}=y_{0}=1.25$.

We solve the problem by the scheme (2.35) with $\Delta t=0.0005, M=128$ and $K=150$. Figure 4 shows the density $\rho(\mathbf{x}, t)=|\psi(\mathbf{x}, t)|^{2}$ and the phase $S(\mathbf{x}, t)$ (with 

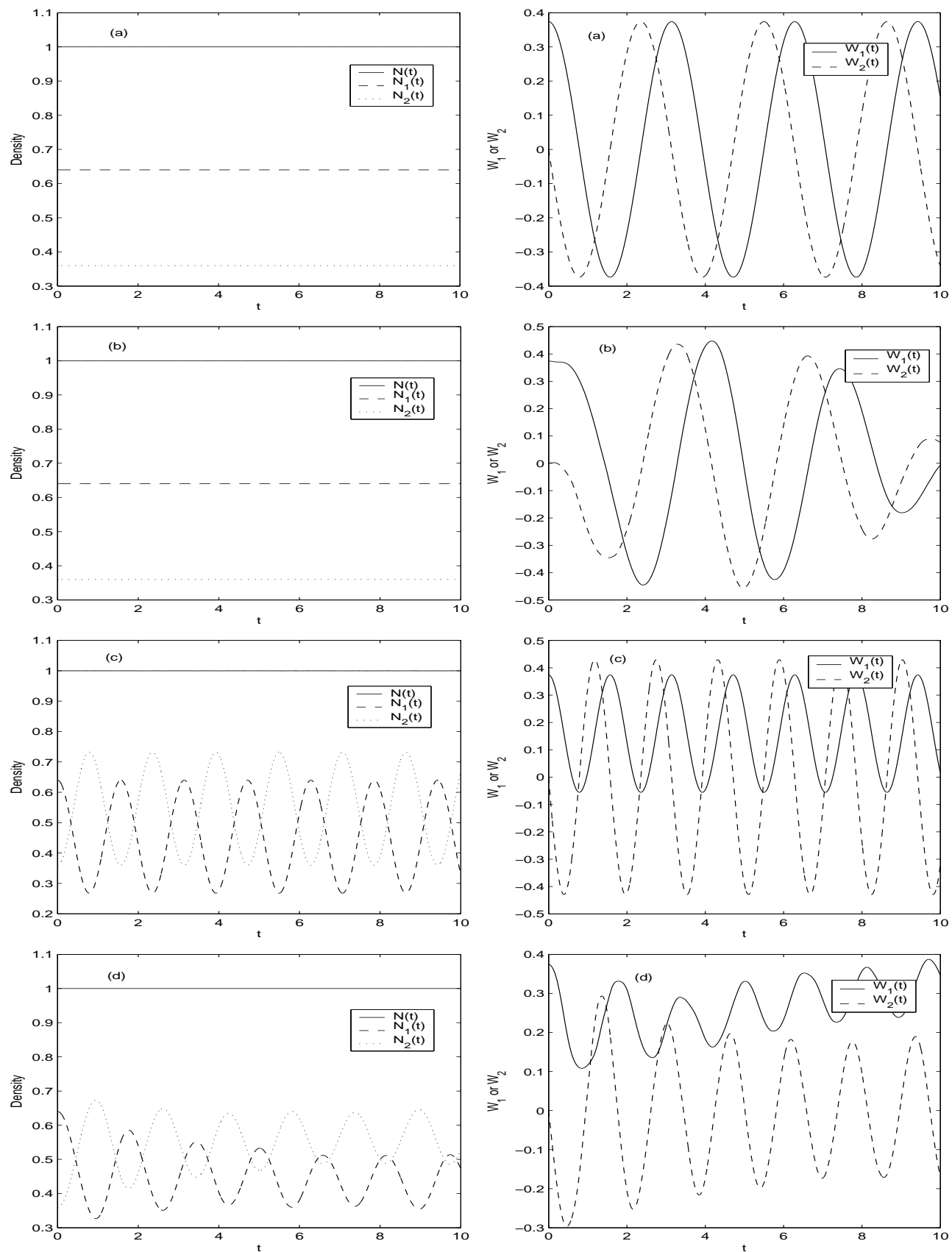

FIG. 3. Time evolution of a few quantities for the dynamics of a rotating two-component BEC in $2 D$ with four sets of parameters: (a) for case (i) $\beta_{11}=\beta_{12}=\beta_{22}=100, \lambda=0$, (b) for case (ii) $\beta_{11}=100, \beta_{12}=80, \beta_{22}=120, \lambda=0$, (c) for case (iii) $\beta_{11}=\beta_{12}=\beta_{22}=100, \lambda=\sqrt{3}$, and (d) for case (iv) $\beta_{11}=100, \beta_{12}=80, \beta_{22}=120, \lambda=\sqrt{3}$.

$\psi=\sqrt{\rho} e^{i S}$ ) of the wave function at different times for case I, and Figure 5 shows similar results for case II. 

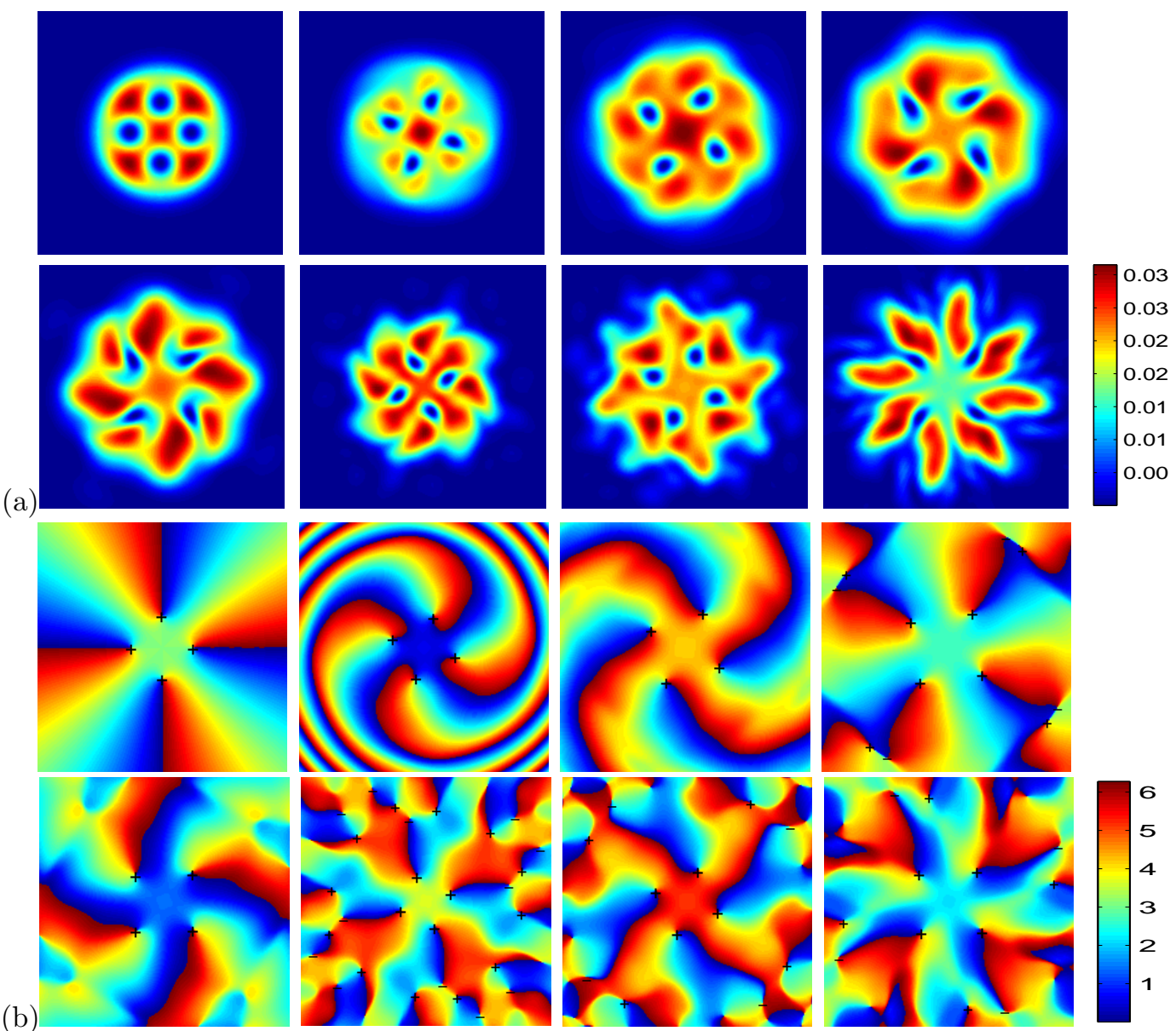

(b)

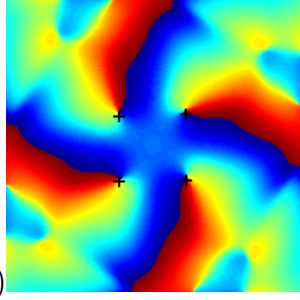

FIG. 4. Contour plots of the density $|\psi(x, y, t)|^{2}$ (row (a)) and phase $S(x, y, t)$ (row (b)) of the wave function (with $\psi=|\psi| e^{i S}$ ) over the dimensionless domain $[-5,5] \times[-5,5]$ for the interaction of two vortex pairs, i.e. Case I, in a rotating BEC in $2 D$ at different times $t=0,0.5,1.0,1.5,2.0,3.0,4.0,5.0$ (in the order of from left to right and from top to bottom).

From Figs. 4\&5, we can draw the following conclusions: (i) In case I, we initially have two vortex pairs with winding number or index $m=1$ and they are located at $\left( \pm x_{0}, 0\right)$ and $\left(0, \pm y_{0}\right)$. When time $t$ evolves, the two vortex pairs rotate clockwise (cf. Fig. 4) and they never collide and annihilate. (ii) In case II, we initially have two vortex dipoles and they are located at $\left( \pm x_{0}, 0\right)$ (with index $m=1$ ) and $\left(0, \pm y_{0}\right)$ (with index $m=-1$ ). When time $t$ evolves, the two vortex dipoles rotate clockwise (cf. Fig. 5 ) and they annihilate simultaneously at $t=t_{1} \leq 3.0$. (iii) In both cases, after $t=t_{0}$ $\left(t_{0}<1.5\right.$ in case I and $t_{0}<1.0$ in case II), several vortex dipoles are generated near the boundary of the condensate and they propagate into the condensate and interact and annihilate with each other (cf. Figs. 4b, 5b). (iv) During the dynamics, vortices are always generated or annihilated in vortex dipoles and thus keep the angular momentum expectation unchanged. This is due to that the trap is radial symmetric and thus the angular momentum expectation is a conserved quantity [5]. (v). From the two cases, we can see that the interaction of vortex pairs and dipoles in rotating BEC can be very interesting and complicated. 

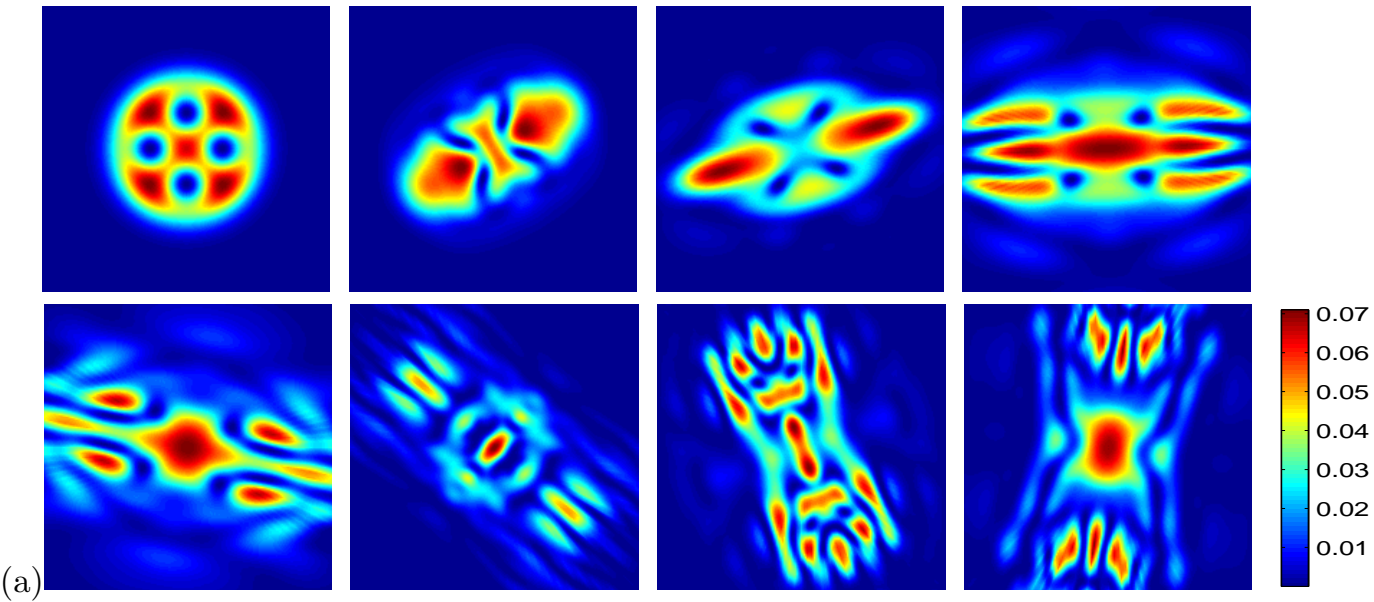

(b)
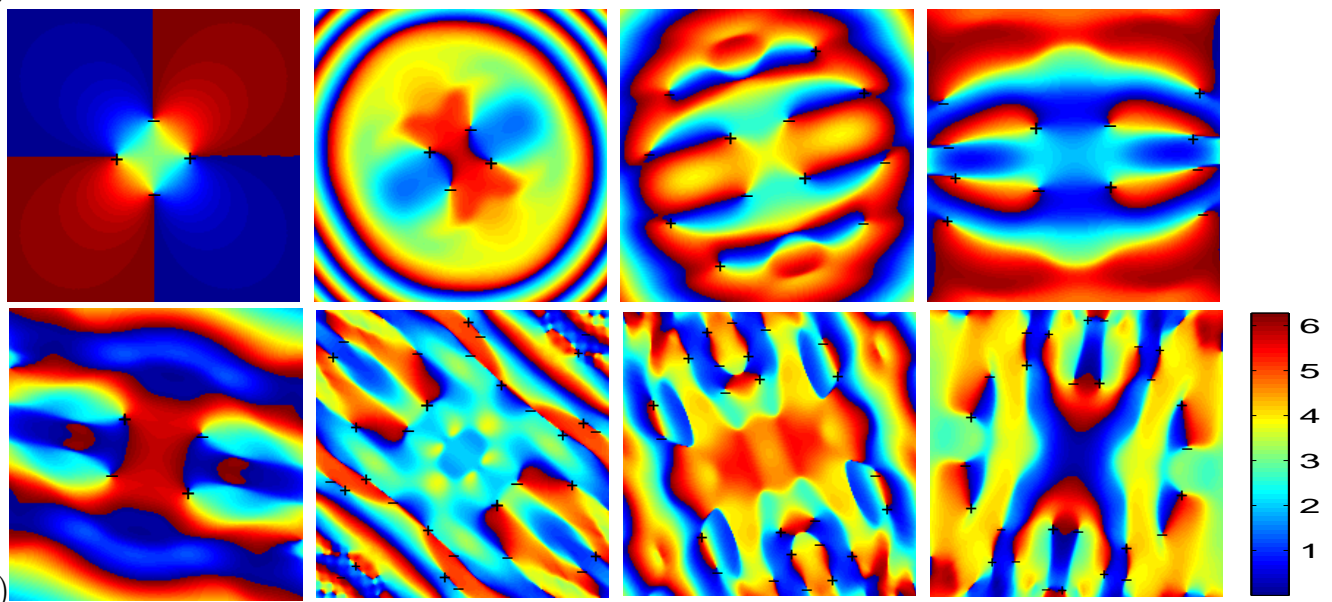

FIG. 5. Contour plots of the density $|\psi(x, y, t)|^{2}$ (row (a)) and phase $S(x, y, t)$ (row (b)) of the wave function over the dimensionless domain $[-5,5] \times[-5,5]$ for the interaction of two vortex dipoles, i.e. Case II, in a rotating BEC in $2 D$ at different times $t=0,0.5,1.0,1.5,2.0,3.0,4.0,5.0$ (in the order of from left to right and from top to bottom).

Example 5. Dynamics of vortex lattices in a rotating BEC in 2D, i.e. we take $d=2$, $\beta_{2}=1000, \Omega=0.9, W(x, y)=\left(\gamma_{y}^{2}-\gamma_{x}^{2}\right) y^{2} / 2$ in (1.1). The initial data in (1.1) is chosen as

$$
\psi_{0}(x, y)=C e^{-\left(x^{2}+y^{2}\right) / 8} \prod_{j=1}^{25} \frac{x-x_{j}+i\left(y-y_{j}\right)}{\sqrt{\left(x-x_{j}\right)^{2}+\left(y-y_{j}\right)^{2}}},
$$

where the constant $C$ is chosen such that $\left\|\psi_{0}\right\|^{2}=\int_{\mathbb{R}^{2}}\left|\psi_{0}(x, y)\right|^{2} d x d y=1$. Here we initially have a vortex lattice consisting of 25 vortices with winding number $m=1$ and their centers uniformly located at a $5 \times 5$ lattice over $[-2,2]^{2}$. We solve the problem by the scheme (2.35) with $\Delta t=0.0001, M=256$ and $K=180$. Figure 6 shows the density $\rho(\mathbf{x}, t)=|\psi(\mathbf{x}, t)|^{2}$ and the phase $S(\mathbf{x}, t)$ of the wave function at different times for $\gamma_{x}=\gamma_{y}=1:=\gamma_{r}$, and Figure 7 shows similar results for $\gamma_{x}=1:=\gamma_{r}$ and $\gamma_{y}=2.0$.

The results in Figs. $6 \& 7$ show that the dynamics of vortex lattice in rotating 

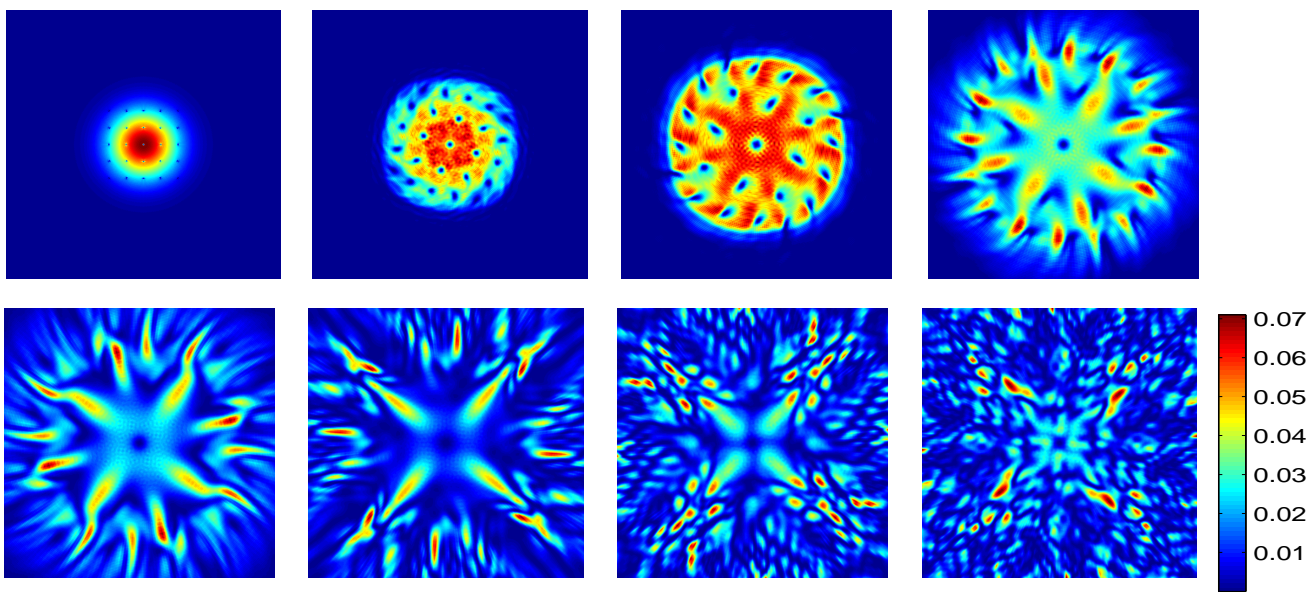

(a)
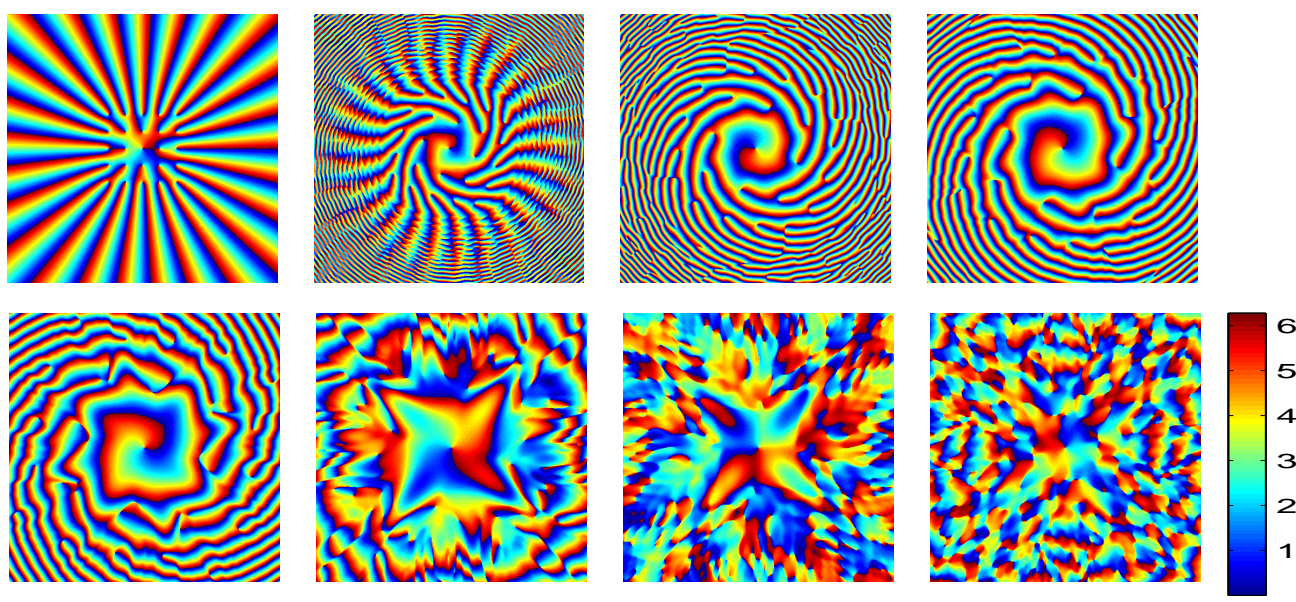

Fig. 6. Contour plots of the density $|\psi(x, y, t)|^{2}$ (row (a)) and phase $S(x, y, t)$ (row (b)) of the wave function over the dimensionless domain $[-8,8] \times[-8,8]$ for the dynamics of a vortex lattice under radial symmetric external trapping, i.e. $\gamma_{x}=\gamma_{y}=1:=\gamma_{r}$ in a rotating BEC in $2 D$ at different times $t=0,0.2,0.4,0.6,0.8,1.2,1.6,2.0$ (in the order of from left to right and from top to bottom).

BEC may be very complicated and interesting and they also demonstrate the high resolution of our method.

5. Concluding remarks. We developed a new generalized-Laguerre-Fourier in $2 \mathrm{D}$, and resp. generalized-Laguerre-Fourier-Hermite in 3D, pseudospectral method to discretize the GPE with an angular momentum rotation term for the dynamics of rotating BEC. The new method adopts polar coordinates in 2D, and resp. cylindrical coordinates in $3 \mathrm{D}$, such that the angular momentum rotation term in the GPE becomes constant coefficient. The new method is based on appropriately scaled generalizedLaguerre, Fourier and Hermite functions and a time-splitting technique to decouple the nonlinearity in the GPE. Hence, it is spectrally accurate in space, second-order or fourth-order accurate in time, explicit, time reversible and time transverse invariant. In addition, the new method has an additional important advantage, i.e. it solves the problem in the whole space instead of in a truncated bounded artificial computational 

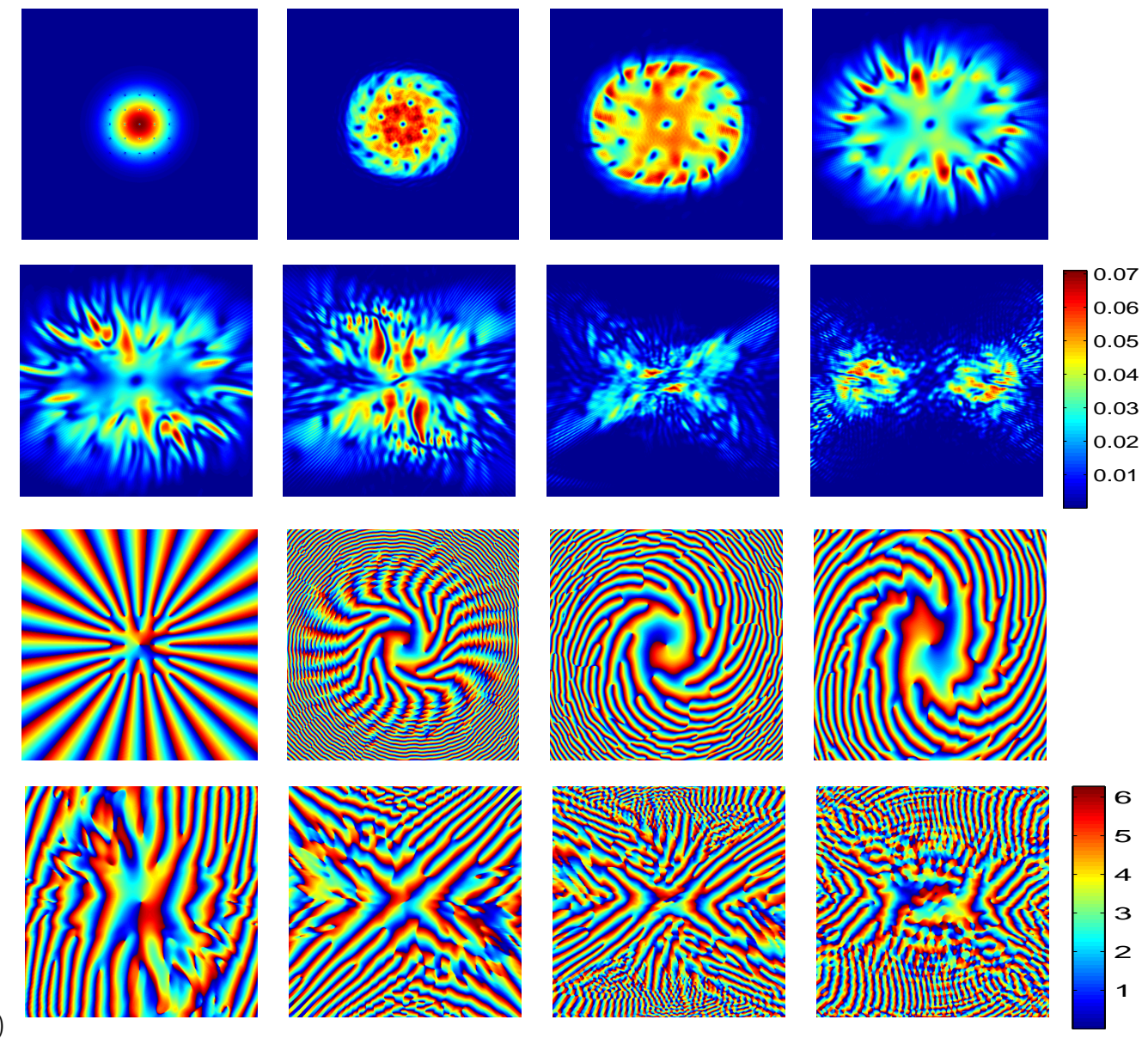

Fig. 7. Contour plots of the density $|\psi(x, y, t)|^{2}$ (row (a)) and phase $S(x, y, t)$ (row (b)) of the wave function over the dimensionless domain $[-8,8] \times[-8,8]$ for the dynamics of a vortex lattice under asymmetric external trapping, i.e. $\gamma_{x}=1:=\gamma_{r}$ and $\gamma_{y}=2.0$, in a rotating BEC in $2 D$ at different times $t=0,0.2,0.4,0.6,0.8,1.2,1.6,2.0$ (in the order of from left to right and from top to bottom).

domain.

\section{REFERENCES}

[1] J. R. Abo-Shaeer, C. Raman, J. M. Vogels and W. Ketterle, Observation of vortex lattices in Bose-Einstein condensates, Science, 292 (2001), pp. 476.

[2] A. Aftalion, Q. Du And Y. Pomeau, Dissipative flow and vortex shedding in the Painlevé boundary layer of a Bose Einstein condensate, Phy. Rev. Lett., 91 (2003), aritcle 090407.

[3] M. H. Anderson, J. R. Ensher, M. R. Matthewa, C. E. Wieman and E. A. Cornell, Observation of Bose-Einstein condensation in a dilute atomic vapor, Science, 269 (1995), pp. 198-201.

[4] W. Bao, Analysis and efficient computation for the dynamics of two-component Bose-Einstein condensates, Contemporary Mathematics, 473 (2008), pp. 1-26.

[5] W. BaO, Q. Du And Y. Zhang, Dynamics of rotating Bose-Einstein condensates and their efficient and accurate numerical computation, SIAM J. Appl. Math., 66 (2006), pp. 758786 
[6] W. Bao, D. Jaksch, And P.A. Markowich, Numerical solution of the Gross-Pitaevskit equation for Bose-Einstein condensation, J. Comput. Phys., 187 (2003), pp. 318-342.

[7] W. BAO, S. Jin AND P. A. MarkowiCH, On time-splitting spectral approximation for the Schrödinger equation in the semiclassical regime, J. Comput. Phys., 175 (2002), pp. 487524 .

[8] W. BaO AND F. Y. Lim, Computing ground states of spin-1 Bose-Einstein condensates by the normalized gradient flow, SIAM J. Sci. Comput., 30 (2008), pp. 1925-1948.

[9] W. Bao, P. A. Markowich, C. Schmeiser and R. M. Weishaupl, On the Gross-Pitaevskii equation with strongly anisotropic confinement: formal asymptotics and numerical experiments, Math. Models Meth. Appl. Sci., 15 (2005), pp. 767-782.

[10] W. BAo AND J. Shen, A Fourth-order time-splitting Laguerre-Hermite pseudo-spectral method for Bose-Einstein condensates, SIAM J. Sci. Comput., 26 (2005), pp. 2010-2028.

[11] W. BAo And J. Shen, A generalized-Laguerre-Hermite pseudospectral method for computing symmetric and central vortex states in Bose-Einstein condensates, J. Comput. Phys., 227 (2008), pp. 9778-9793.

[12] W. BAO AND H. WANG, An efficient and spectrally accurate numerical method for computing dynamics of rotating Bose-Einstein condensates, J. Comput. Phys., 217 (2006), pp. 612626.

[13] W. BAO, H. WANG AND P.A. MARKOWICH, Ground, symmetric and central vortex states in rotating Bose-Einstein condensates, Commun. Math. Sci., 3 (2005), pp. 57-88.

[14] B. M. Caradoc-Davis, R. J. Ballagh, K. Burnett, Coherent dynamics of vortex formation in trapped Bose-Einstein condensates, Phys. Rev. Lett., 83 (1999), pp. 895-898.

[15] Y. Castin, Z. Hadzibabic, S. Stock, J. Dalibard and S. Stringari, Quantized vortices in the ideal bose gas: A physical realization of random polynomials, Phys. Rev. Lett., 96 (2006) article 040405.

[16] K. B. Davis, M. O. Mewes, M. R. Andrews, N. J. van Druten, D. S. Durfee, D. M. Kurn And W. Ketterle, Bose-Einstein condensation in a gas of sodium atoms, Phys. Rev. Lett., 75 (1995), pp. 3969-3973.

[17] D. L. Feder, C. W. Clark AND B. I. Schneider, Nucleation of vortex arrays in rotating anisotropic Bose-Einstein condensates, Phys. Rev. A, 61 (1999), article 011601.

[18] D. L. Feder, C. W. Clark and B. I. Schneider, Vortex stability of interacting Bose-Einstein condensates confined in anisotropic harmonic traps, Phys. Rev. Lett., 82 (1999), pp. 49564959.

[19] B.-Y. GUO AND J. SHEN, Laguerre-Galerkin method for nonlinear partial differential equations on a semi-infinite interval, Numer. Math., 86 (2000), pp. 635-654.

[20] B.-Y. GuO AND X.-Y. Zhang, A new generalized Laguerre spectral approximation and its applications, J. Comput. Appl. Math., 181 (2005), pp. 342-363.

[21] P. C. Haljan, I. Coddington, P. Engles And E. A. Cornell, Driving Bose-Einsteincondensate vorticity with a rotating normal cloud, Phys. Rev. Lett., 87 (2001), article 210403.

[22] C. C. HaO, L. HsiaO AND H. L. Li, Global well posedness for the Gross-Pitaevskii equation with an angular momentum rotational term in three dimensions, J. Math. Phys., 48 (2007), article 102105.

[23] T. L. Ho, Spinor Bose condensates in optical traps, Phys. Rev. Lett., 81 (1998), pp. 742-745.

[24] B. Jackson, J. F. MCCAnn And C. S. AdAms, Vortex formation in dilute inhomogeneous Bose-Einstein condensates, Phys. Rev. Lett., 80 (1998), pp. 3903-3906.

[25] K. T. Kapale And J. P. Dowling, Vortex phase qubit: Generating arbitrary, counterrotating, coherent superpositions in bose-einstein condensates via optical angular momentum beams, Phys. Rev. Lett., 95 (2005) 173601.

[26] K. Kasamatsu, M. Tsubota And M. Ueda, Nonlinear dynamics of vortex lattice formation in a rotating Bose- Einstein condensate, Phys. Rev. A, 67 (2003), article 033610.

[27] I. K. Khabibrakhmanov and D. Summers, The use of generalized Laguerre polynomials in spectral methods for nonlinear differential equations, Comput. Math. Appl., 36 (1998), pp. 65-70.

[28] A. Klein, D. Jaksch, Y. Zhang And W. BaO, Dynamics of vortices in weakly interacting Bose-Einstein condensates, Phys. Rev. A, 76 (2007) article 043602.

[29] A. E. Leanhardt, A. Gorlitz, A. P. Chikkatur, D. Kielpinski, Y. Shin, D. E. Pritchard AND W. KeTtERLE, Imprinting vortices in a Bose-Einstein condensate using topological phases, Phys. Rev. Lett., 89 (2002) article 190403.

[30] A. E. Leanhardt, Y. Shin, D. Kielpinski, D.E. Pritchard and W. Ketterle, Coreless vortex formation in a spinor Bose-Einstein condensate, Phys. Rev. Lett., 90 (2003), article 140403. 
[31] H. Ma, W. Sun And T. TANG, Hermite spectral methods with a time-dependent scaling for parabolic equations in unbounded domains, SIAM J. Numer. Anal., 43 (2005), pp. 58-75.

[32] K.W. Madison, F. Chevy, W. Wohlleben and J. Dalibard, Vortex formation in a stirred Bose-Einstein condensate, Phys. Rev. Lett., 84 (2000), pp. 806-809.

[33] K. W. Madison, F. Chevy, V. Bretin and J. Dalibard, Stationary states of a rotating Bose-Einstein condensate: routes to vortex nucleation, Phys. Rev. Lett., 86 (2001), pp. 4443-4446.

[34] M.R. Matthews, B.P. Anderson, P.C. Haljan, D.S. Hall, C.E. Wiemann and E.A. Cornell, Vortices in a Bose-Einstein condensates, Phys. Rev. Lett., 83 (1999), pp. 24982501.

[35] A. Minguzzi, S. Succi, F. Toschi, M. P. Tosi, P. Vignolo, Numerical methods for atomic quantum gases with applications to Bose-Einstein condensates and to ultracold fermions, Phys. Rep., 395 (2004), pp. 223-355.

[36] L.P. Pitaevskit and S. Stringari, Bose-Einstein condensation, Clarendon Press, 2003.

[37] C. Raman, J. R. Abo-Shaeer, J. M. Vogels, K. Xu and W. Ketterle, Vortex nucleation in a stirred Bose-Einstein condensate, Phys. Rev. Lett., 87 (2001), article 210402.

[38] D.S. RokhSAR, Vortex stability and persistent currents in trapped Bose-gas, Phys. Rev. Lett., 79 (1997), pp. 2164-2167.

[39] J. SHen, Stable and efficient spectral methods in unbounded domains using Laguerre functions, SIAM J. Numer. Anal., 38 (2000), pp. 1113-1133.

[40] G. Szegö, Orthogonal Polynomials 4th ed., Amer. Math. Soc. Colloq. Publ. 23, AMS, Providence, RI, 1975.

[41] G. Strang, On the construction and comparison of difference schemes, SIAM J. Numer. Anal., 5 (1968), pp. 505-517.

[42] T. TANG, The Hermite spectral method for Gaussian-type functions, SIAM J. Sci. Comput., 14 (1993), pp. 594-606.

[43] M. Thalhammer, High-order exponential operator splitting methods for time-dependent Schrödinger equations, SIAM J. Numer. Anal., 46 (2007), pp. 2022-2038.

[44] J. E. Williams and M. J. Hoonnd, Preparing topological states of a Bose-Einstein condensate, Nature, 401 (1999), pp. 568.

[45] H. Yoshida, Construction of higher order symplectic integrators, Phys. Lett. A, 150 (1990), pp. 262-268.

[46] Y. Zhang, W. Bao and H. Li, Dynamics of rotating two-component Bose-Einstein condensates and its efficient computation, Physica D, 234 (2007), pp. 49-69. 